\title{
SCALAR EXTENSION OF BICOALGEBROIDS
}

\author{
IMRE BÁLINT
}

\begin{abstract}
After recalling the definition of a bicoalgebroid, we define comodules and modules over a bicoalgebroid. We construct the monoidal category of comodules, and define Yetter-Drinfel'd modules over a bicoalgebroid. It is proved that the Yetter-Drinfel'd category is monoidal and pre-braided just as in the case of bialgebroids, and is embedded into the one-sided center of the comodule category. We proceed to define Braided Cocommutative Coalgebras (BCC) over a bicoalgebroid, and dualize the scalar extension construction of [2] and 1], originally applied to bialgebras and bialgebroids, to bicoalgebroids. A few classical examples of this construction are given. Identifying the comodule category over a bicoalgebroid with the category of coalgebras of the associated comonad, we obtain a comonadic (weakened) version of Schauenburg's theorem. Finally, we take a look at the scalar extension and braided cocommutative coalgebras from a (co-)monadic point of view.
\end{abstract}

\section{INTRODUCTION}

Bicoalgebroids were introduced by Brzeziński and Militaru in 2 as the structure that dualizes bialgebroids (in fact, Takeuchi's $\times_{R}$-bialgebras) in the sense of reversing arrows. This notion is not to be confused with the different kinds of bialgebroid-duals that were later introduced in [8]. It would seem that the study of bicoalgebroids hasn't been taken up vigorously since their inception; in our view, they merit attention for at least two reasons. First, it is well established that a bialgebroid may be thought of as a non-commutative analogue of the algebra of functions on a groupoid. It follows that a bicoalgebroid, in turn, should be regarded as a non-commutative analogue of the groupoid itself. This raises the hope that classical constructions on groupoids may find their non-commutative generalizations more easily in the context of bicoalgebroids. Secondly, just as bialgebroids play a fundamental role in depth-two extensions of algebras, it is expected that bicoalgebroids feature prominently in extensions of coalgebras (from a different approach, in [9] Kadison constructs bialgebroids from depth 2 extensions of coalgebras). To complete the picture, the dual Hopf-Galois theory of [16] for extensions of coalgebras should generalize (from bialgebras) to bicoalgebroids, giving a dual version of bialgebroid-Galois theory. Further work in this latter direction is deferred to a subsequent publication.

Central to this paper is the introduction of scalar extension for bicoalgebroids. Incidentally, the construction that was shown in [1] to be a non-commutative version of scalar extension was defined (for Hopf-algebras) in [2 - alongside with bicoalgebroids. 


\section{BiCOALGEBRoIds; COMODUles AND MODUlES}

Throughout, $k$ will be a field and the category $\mathrm{M}=\mathrm{M}_{k}$ of $k$-modules will serve as our underlying category. The unadorned $\otimes$ always means $\otimes_{k}$.

We use the ubiquitous Sweedler notation for coproducts and coactions. For a coalgebra $\langle C, \Delta, \varepsilon\rangle$, the coproduct $\Delta: C \rightarrow C \otimes C$ on elements is denoted $\Delta(c)=$ $c_{(1)} \otimes c_{(2)}$, with an implicit finite summation understood, i.e. $c_{(1)} \otimes c_{(2)}=\sum_{i} c_{(1)}{ }^{i} \otimes$ $c_{(2)}{ }^{i}$. Quite similarly, a right $C$-coaction $\rho_{M}: M \rightarrow M \otimes C$ will be denoted $\rho_{M}(m)=m_{[0]} \otimes m_{[1]}$ and a left $C$-coaction $\lambda_{N}: N \rightarrow C \otimes N$ will be denoted $\lambda_{N}(n)=n_{[-1]} \otimes n_{[0]}$.

The category of bicomodules over a $k$-coalgebra $C$ is monoidal with monoidal unit $C$ and monoidal product the cotensor product over $C$. This category will be referred to as $\left\langle{ }^{C} \mathrm{M}^{C}, \square_{C}, C\right\rangle$.

In fact, if $C$ is a coalgebra over a ring $R$ which is flat as an $R$-module, then the category of $R$-flat $C$-bicomodules is monoidal with monoidal product the cotensor product over $C$, and monoidal unit $C$.

We shall also use the following standard notations throughout the paper. The coopposite coalgebra of a coalgebra $\langle C, \Delta, \varepsilon\rangle$ is $C_{c o p}=\left\langle C, \Delta_{c o p}, \varepsilon\right\rangle$, with the coproduct $\Delta_{\text {cop }}(c)=\operatorname{tw}_{C, C} \circ \Delta(c)=c_{(2)} \otimes c_{(1)}$. In analogy to the concept of enveloping algebra, the co-enveloping coalgebra of $C$ is $C^{e}=\left\langle C \otimes C_{c o p}, \operatorname{tw}_{23} \circ\left(\Delta \otimes \Delta_{c o p}\right), \varepsilon \otimes \varepsilon\right\rangle$.

Following [2], we recall the following (somewhat lengthy)

Definition 2.1. A left bicoalgebroid $\langle H, \Delta, \varepsilon, \mu, \eta, \alpha, \beta, C\rangle$ consists of

- a $k$-coalgebra $\left\langle H, \Delta_{H}, \varepsilon_{H}\right\rangle$

- two coalgebra maps $\alpha: H \rightarrow C$ and $\beta: H \rightarrow C_{c o p}$, such that $\alpha$ and $\beta$ 'cocommute', i.e. $\alpha\left(h_{(1)}\right) \otimes \beta\left(h_{(2)}\right)=\alpha\left(h_{(2)}\right) \otimes \beta\left(h_{(1)}\right)$. These maps furnish $H$ with a $(C \otimes C)$-bicomodule structure, such that $\left(H ; \lambda_{L}, \lambda_{R} ; \rho_{L}, \rho_{R}\right) \in$ $C \otimes C \mathrm{M}^{C \otimes C}$. The four $C$-coactions are:

$$
\begin{array}{ll}
\lambda_{L}(h)=\alpha\left(h_{(1)}\right) \otimes h_{(2)}, & \rho_{L}(h)=h_{(2)} \otimes \beta\left(h_{(1)}\right) \\
\lambda_{R}(h)=\beta\left(h_{(2)}\right) \otimes h_{(1)}, & \rho_{R}(h)=h_{(1)} \otimes \alpha\left(h_{(2)}\right)
\end{array}
$$

- $C$-bicomodule maps $\mu_{H}: H \square_{C} H \rightarrow H$ and $\eta_{H}: C \rightarrow H$ (multiplication \& unit) making $\left(H, \lambda_{L}, \rho_{L}\right)$ an algebra in ${ }^{C} \mathrm{M}^{C}$,

subject to the following axioms:

(1) The multiplication map $\mu: H \square_{C} H \rightarrow H$ satisfies:

$$
\sum_{i} \mu\left(g^{i} \otimes h_{(1)}^{i}\right) \otimes \alpha\left(h_{(2)}^{i}\right)=\mu\left(g_{(1)}^{i} \otimes h^{i}\right) \otimes \beta\left(g_{(2)}^{i}\right)
$$

(2) and it is comultiplicative:

$$
\Delta \circ \mu\left(\sum_{i} g^{i} \otimes h^{i}\right)=\sum_{i} \mu\left(g_{(1)}^{i} \otimes h_{(1)}^{i}\right) \otimes \mu\left(g_{(2)}^{i} \otimes h_{(2)}^{i}\right)
$$

(3) Furthermore, the product is counital (note that this axiom seems to be missing in Ref. [2]):

$$
\varepsilon(g) \varepsilon(h)=\varepsilon \circ \mu(g \otimes h)
$$

(4) The unit map $\eta: C \rightarrow H$ satisfies the unit axiom:

$$
\mu \circ(\eta \square H) \circ \lambda_{L}=H=\mu \circ(H \square \eta) \circ \rho_{L}
$$


(5) The unit map is compatible with the coalgebra structure in the following sense:

$$
\begin{gathered}
\Delta(\eta(c))=\eta(c)_{(1)} \otimes \eta\left(\alpha\left(\eta(c)_{(2)}\right)\right)=\eta(c)_{(1)} \otimes \eta\left(\beta\left(\eta(c)_{(2)}\right)\right) \\
\varepsilon(\eta(c))=\varepsilon(c)
\end{gathered}
$$

In [2], it is first proved that the condition 2.1 makes sense, i.e. the two sides of the equation are well defined maps. This, in turn, implies that 2.2 makes sense, which boils down to $(\mu \square \mu) \circ \operatorname{tw}_{23} \circ(\Delta \square \Delta)$ being a well-defined map. The condition 2.1 on the multiplication map may be rephrased by saying that $\mu$ factorizes through the cocenter of the $C$-bicomodule ${ }^{C} H \square H^{C}$, where the two coactions are $\lambda_{R}$ and $\rho_{R}$. We define the cocenter for bicomodules as follows.

Definition 2.2. Let $M \in{ }^{C} \mathrm{M}^{C}$ a $C$-bicomodule. Define the map

$$
\begin{aligned}
\Phi: M \otimes C^{*} & \rightarrow M \\
m \otimes \varphi & \mapsto m_{[0]} \varphi\left(m_{[1]}\right)-m_{[0]} \varphi\left(m_{[-1]}\right)
\end{aligned}
$$

where $C^{*}$ denotes the $k$-dual of the coalgebra $C$. Then, the cocenter of $M$ is defined by the cokernel map $\zeta: M \rightarrow \mathcal{Z}(M)$, where

$$
M \otimes C^{*} \stackrel{\Phi}{\longrightarrow} M \stackrel{\zeta}{\longrightarrow} \mathcal{Z}(M)
$$

Introduce also the epi-mono factorization $\Phi: M \otimes C^{*} \stackrel{e}{\rightarrow} J_{M} \stackrel{i}{\rightarrow} M$

The cocenter satisfies the following universal property. Let

$$
W_{M}=\left\{m_{[0]} \otimes m_{[1]}-m_{[0]} \otimes m_{[-1]} \mid m \in M\right\} \subset M \otimes C
$$

then for all $k$-module maps $f: M \rightarrow N$ which satisfy

$$
(f \otimes C)\left(W_{M}\right)=0
$$

i.e. $f\left(m_{[0]}\right) \otimes m_{[1]}=f\left(m_{[0]}\right) \otimes m_{[-1]}$, there is a unique $f^{\prime}: \mathcal{Z}(M) \rightarrow N$ such that $f=f^{\prime} \circ \zeta$ :

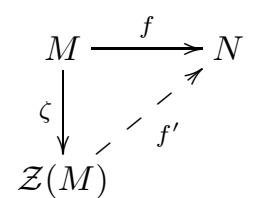

Indeed, applying $(N \otimes \varphi)$ to 2.7 we find that $(f \otimes \varphi)\left(W_{M}\right)=0$ for all $\varphi \in C^{*}$, i.e. $f$ annihilates $J_{M}$.

If the coalgebra $C$ is locally projective as a $k$-module (see $[3,42.9$ ), then $(\zeta \otimes C)\left(W_{M}\right)=0$. To see this, note that for $C$ locally projective, $(\zeta \otimes C)\left(W_{M}\right)=0$ if and only if $(I d \otimes \varphi) \circ(\zeta \otimes C)\left(W_{M}\right)=0$ for all $\varphi \in C^{*}$. This, however, holds by the definiton of $\zeta$.

Thus, for locally projective $C$, a $k$-module map $f: M \rightarrow N$ factorizes through $\zeta: M \rightarrow \mathcal{Z}(M)$ if and only if $(f \otimes C)\left(W_{M}\right)=0$. Since, throughout this paper, we are working over a field, it is in fact unnecessary to explicitly assume local projectivity: modules over a field are always free, hence they are projective. A projective module is also locally projective. 
We apply the above definition to the bicomodule ${ }^{C} H \square H^{C}$ afforded by the coactions $\lambda_{R}$ and $\rho_{R}$. For reference, the bicomodule structure is

$$
\begin{aligned}
& \left(\lambda_{R} \square H\right): \sum_{i} g^{i} \square h^{i} \mapsto \sum_{i} \beta\left(g_{(2)}^{i}\right) \square g_{(1)}^{i} \square h^{i} \\
& \left(H \square \rho_{R}\right): \sum_{i} g^{i} \square h^{i} \mapsto \sum_{i} g^{i} \square h_{(1)}^{i} \square \alpha\left(h_{(2)}^{i}\right)
\end{aligned}
$$

We make the following

Definition 2.3. $H \otimes H$ is the cocenter of the bicomodule ${ }^{C} H \square H^{C}$,

$$
H \square_{C} H \otimes C^{*} \stackrel{\Phi_{2}}{\longrightarrow} H \square_{C} H \stackrel{\zeta_{2}}{\longrightarrow} H \otimes H
$$

where $\Phi_{2}\left(g^{i} \square h^{i} \otimes \varphi\right)=g^{i} \square h_{(1)}^{i} \varphi\left(\alpha\left(h_{(2)}^{i}\right)\right)-g_{(1)}^{i} \square h^{i} \varphi\left(\beta\left(g_{(2)}^{i}\right)\right)$

Using 2.8 and 2.9. the multiplication map $\mu: H \square_{C} H \rightarrow H$ factorizes through $H \otimes H$, i.e.

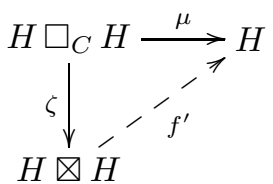

precisely if $\sum_{i} \mu\left(g^{i} \otimes h_{(1)}^{i}\right) \otimes \alpha\left(h_{(2)}^{i}\right)=\mu\left(g_{(1)}^{i} \otimes h^{i}\right) \otimes \beta\left(g_{(2)}^{i}\right)$ (condition 2.1) holds.

This construction can be seen as dual to that of the Takeuchi product $\times_{R}$. For a left bialgebroid $A$, the submodule $A \times_{R} A \hookrightarrow A \otimes_{R} A$ is the center of the $R$-bimodule $r \cdot(A \otimes A) \cdot r^{\prime}=A t(r) \otimes A s(r)$. It is well-known that there is no well-defined multiplication on $A \otimes A$, but $A \times_{R} A$ is a ring with component-wise multiplication. The dual result is that even though comultiplication is not well-defined on $H \square_{C} H$, the factor $H \otimes H$ becomes a well-defined coalgebra. This ensures that 2.2 is well-defined.

The reader may easily convince herself that these axioms are dual to those of a left bialgebroid $\left\langle A, \mu_{A}, \eta_{A}, \Delta_{A}, \varepsilon_{A}, s, t, R\right\rangle$ in the sense of reversing arrows and making the following substitutions: $\left\langle A, \mu_{A}, \eta_{A}\right\rangle \leftrightarrow\left\langle H, \Delta_{H}, \varepsilon_{H}\right\rangle,\left\{\Delta_{A}, \varepsilon_{A}\right\} \leftrightarrow\left\{\mu_{H}, \eta_{H}\right\}$, $\{s, t\} \leftrightarrow\{\alpha, \beta\}, R \leftrightarrow C$.

A right bicoalgebroid is a $C$-bicomodule algebra with the coactions $\lambda_{R}$ and $\rho_{R}$, i.e. we require $\left(H, \lambda_{R}, \rho_{R}\right)$ to be a monoid in the category of $C$-bicomodules. The axioms dualize those of a right bialgebroid (cf. the Lemma below).

We note here a result in line with the duality between bialgebroids and bicoalgebroids. It is well-known that the simplest right bialgebroid over a ring $R$ is the enveloping algebra $R^{e}=R \otimes R^{o p}$ (its opposite is a left bialgebroid). The following, dual statement provides our first example of a bicoalgebroid:

Lemma 2.4. The co-enveloping coalgebra $C^{e}=C \otimes C_{c o p}$ is a right bicoalgebroid with the following structure maps. The source- and target maps are given by

$$
\alpha: C \otimes C_{c o p} \rightarrow C, c \otimes \bar{c} \mapsto c \varepsilon(\bar{c}) \text { and } \beta: C \otimes C_{c o p} \rightarrow C_{c o p}, c \otimes \bar{c} \mapsto \varepsilon(c) \bar{c}
$$

Multiplication is

$$
\mu^{e}: C \otimes C_{c o p} \square C \otimes C_{c o p} \rightarrow C \otimes C_{c o p}, c \otimes \bar{c} \square d \otimes \bar{d} \mapsto d \varepsilon(c) \varepsilon(\bar{d}) \otimes \bar{c}
$$

and the unit map is $\Delta_{c o p}, \eta^{e}: C \rightarrow C \otimes C_{c o p}, \eta^{e}(c)=c_{(2)} \otimes c_{(1)}$. 
Proof. $C^{e}$ has the (right-bicoalgebroid type) $C$-bicomodule structure coming from $\lambda_{R}=\left(\beta \otimes C^{e}\right) \circ \Delta_{c o p}$ and $\rho_{R}=\left(C^{e} \otimes \alpha\right) \circ \Delta$. Explicity, the coactions are

$$
\begin{aligned}
& \lambda_{R}(c \otimes \bar{c})=\bar{c}_{(1)} \otimes\left(c \otimes \bar{c}_{(2)}\right) \\
& \rho_{R}(c \otimes \bar{c})=\left(c_{(1)} \otimes \bar{c}\right) \otimes c_{(2)}
\end{aligned}
$$

Multiplication and unit are then seen to be (left and right) $C$-bicomodule maps with respect to the coactions $\lambda_{R}$ and $\rho_{R}$. Associativity and the unit property are easy calculations. $c \otimes \bar{c} \square d \otimes \bar{d} \in C \otimes C_{c o p} \square_{C} C \otimes C_{c o p}$ means

$$
\left(c_{(1)} \otimes \bar{c}\right) \otimes c_{(2)} \otimes d \otimes \bar{d}=(c \otimes \bar{c}) \otimes \bar{d}_{(1)} \otimes\left(d \otimes \bar{d}_{(2)}\right)
$$

We have to prove that multiplication factorizes through $C^{e} \otimes C^{e}$, i.e. $\alpha\left((c \otimes \bar{c})_{(1)}\right) \otimes$ $\mu\left((c \otimes \bar{c})_{(2)} \square(d \otimes \bar{d})\right)=\beta\left((d \otimes \bar{d})_{(1)}\right) \otimes \mu\left((c \otimes \bar{c}) \otimes(d \otimes \bar{d})_{(2)}\right)$. Inserting definitions, this reads

$$
c_{(1)} \otimes \mu^{e}\left(\left(c_{(2)} \otimes \bar{c}\right) \square(d \otimes \bar{d})\right)=\bar{d}_{(2)} \otimes \mu^{e}\left((c \otimes \bar{c}) \square\left(d \otimes \bar{d}_{(1)}\right)\right)
$$

By the definition of $\mu^{e}$, 2.11 simplifies to

$$
c \otimes d \varepsilon(\bar{d}) \otimes \bar{c}=\bar{d} \otimes d \varepsilon(c) \otimes \bar{c}
$$

which is a consequence of 2.10 (applying the counit map twice). It now makes sense to demand the compatibility of multiplication and comultiplication,

$$
\begin{aligned}
& \left(\mu^{e} \otimes \mu^{e}\right) \circ \mathrm{tw}_{2,3} \circ\left(\Delta^{e} \otimes \Delta^{e}\right)\left((c \otimes \bar{c})((d \otimes \bar{d}))=\left(c_{(1)} \otimes \bar{c}_{(2)}\right)\left(d_{(1)} \otimes \bar{d}_{(2)}\right) \otimes\right. \\
& \otimes\left(c_{(2)} \otimes \bar{c}_{(2)}\right)\left(d_{(2)} \otimes \bar{d}_{(1)}\right)=\left(d_{(1)} \varepsilon(c) \varepsilon(\bar{d}) \otimes \bar{c}_{(2)}\right) \otimes\left(d_{(2)} \otimes \bar{c}_{(1)}\right)= \\
& =\Delta^{e} \circ \mu^{e}((c \otimes \bar{c}) \otimes(d \otimes \bar{d}))
\end{aligned}
$$

We skip the proof of the remaining compatibilities, all of them being trivial calculations.

Just as in the dual case (where $R^{e, o p}=R^{o p} \otimes R$ is a left bialgebroid), we also have that $C_{c o p}^{e}=C_{c o p} \otimes C$ is a left bicoalgebroid. The proof is entirely similar.

2.1. Comodules over a bicoalgebroid. Based on experience with bialgebroids and dualization arguments, it may be expected that a more categorical approach to bicoalgebroids leads to the study of it's category of comodules.

It is a well-known fact that a coalgebra map $\gamma: D \rightarrow C$ induces a $C$-bicomodule structure on $D$ such that $D$ becomes a comonoid in ${ }^{C} \mathrm{M}^{C}$. The category of $D^{-}$ comodules is then naturally constructed as a subcategory of ${ }^{C} \mathrm{M}^{C}$. We specialize this remark to the case of a (left-) bicoalgebroid $H$ over $C$. Consider the coalgebra map $\varphi=(\alpha \otimes \beta) \circ \Delta: H \rightarrow C \otimes C_{c o p}$. The left and right $C^{e}$-coaction induced by $\varphi$ on $H$ are $\lambda=(\varphi \otimes H) \circ \Delta$ and $\rho=(H \otimes \varphi) \circ \Delta$. Inserting the definition of $\varphi$ and comparing with the notation of 2.1 .

$$
\begin{aligned}
& \lambda=\left(C \otimes \rho_{L}^{o p}\right) \circ \lambda_{L}: H \rightarrow\left(C \otimes C_{c o p}\right) \otimes H \\
& \rho=\left(\rho_{R} \otimes C\right) \circ \lambda_{R}^{o p}: H \rightarrow H \otimes\left(C \otimes C_{c o p}\right)
\end{aligned}
$$

(by $\lambda_{R}^{o p}$, for example, we mean the right $C_{c o p}$-coaction corresponding to $\lambda_{R}$ through the isomorphism $\left.{ }^{C} \mathrm{M} \simeq \mathrm{M}^{C_{c o p}}\right)$. The image of $\Delta_{H}$ lies in $H \square_{C e} H$, i.e. we can introduce the $C^{e}$-bicomodule map $\bar{\Delta}: H \rightarrow H \square_{C^{e}} H$ with

$$
\Delta_{H}: H \stackrel{\bar{\Delta}}{\longrightarrow} H \square_{C^{e}} H \stackrel{\bar{\iota}_{H, H}}{\longrightarrow} H \otimes H
$$


(here, $\bar{\iota}_{M, N}: M \square_{C^{e}} N \rightarrow M \otimes N$ is the equalizer defining the cotensor product over $C^{e}$ ). On the other hand, the $C^{e}$-bicomodule map $\varphi$ is the composite

$$
\varepsilon_{H}: H \stackrel{\varphi}{\longrightarrow} C^{e} \stackrel{\varepsilon \otimes \varepsilon}{\longrightarrow} k
$$

since $\varphi$ is a coalgebra map. It is then straightforward to show that

Lemma 2.5. $\langle H, \bar{\Delta}, \bar{\varepsilon}=\varphi\rangle$ is a comonoid in ${ }^{C} \mathrm{M}^{C^{e}}$.

Proof. Coassociativity is trivial, and the counit property reads

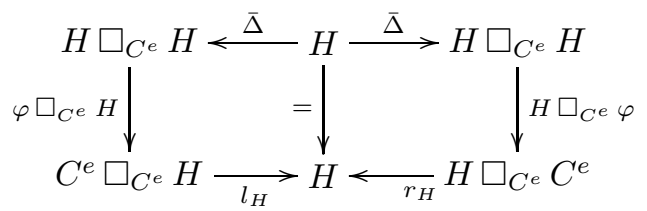

which commutes, because $(\varepsilon \otimes \varepsilon) \circ\left(\varphi\left(h_{(1)}\right)\right) h_{(2)}=\varepsilon_{H}\left(h_{(1)}\right) h_{(2)}=h$, etc.

We can now define the category of comodules over $H$.

Definition 2.6. A left $H$-comodule over a left bicoalgebroid $H$ is a pair $\left\langle M, \delta_{M}\right\rangle$, where $M \in{ }^{e} \mathrm{M}^{C^{e}}$, and $\delta_{M}: M \rightarrow H \square_{C^{e}} M$ is a $C^{e}$-bicomodule map for which
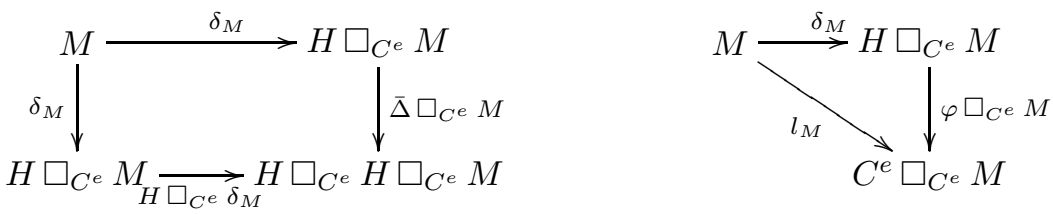

making $\delta_{M}$ a coassociative \& counital coaction.

The category of $H$-comodules ${ }^{H} \mathrm{M}$ has objects the left $H$-comodules, and the arrows $f:\left\langle M, \delta_{M}\right\rangle \rightarrow\left\langle N, \delta_{N}\right\rangle$ are the $C^{e}$-bicomodule maps $f: M \rightarrow N$ such that

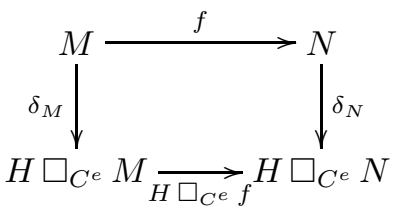

Summarizing, a left bicoalgebroid $H$ over $C$ is simultaneously a monoid in the category ${ }^{C} \mathrm{M}^{C}$ (with coactions $\left(H, \lambda_{L}, \rho_{L}\right)$ ) and a comonoid in the category ${ }^{C} \mathrm{M}^{C^{e}}$ (with coactions $(H, \lambda, \rho)$ ). This phenomenon is already familiar from the theory of bialgebroids, namely that the algebra and coalgebra structures live in different monoidal categories.

The forgetful functor associated to the map $\varphi: H \rightarrow C^{e}$,

$$
\begin{aligned}
& F:{ }^{H} \mathrm{M} \rightarrow{ }^{C^{e}} \mathrm{M} \simeq{ }^{C} \mathrm{M}^{C} \\
& \left\langle M, \delta_{M}\right\rangle \rightarrow\left\langle M,(\varphi \otimes M) \circ \delta_{M}\right\rangle
\end{aligned}
$$

is faithful and left adjoint to $H \square_{C^{e}}:{ }^{C^{e}} \mathrm{M} \rightarrow{ }^{H} \mathrm{M}$. Let us briefly recall the dual situation: a left bialgebroid $A$ over $R$ is an $R^{e}$-ring with $s \otimes t: R \otimes R^{o p} \rightarrow A$, i.e. a monoid in $R_{R^{e}} \mathrm{M}_{R^{e}}$. The forgetful functor $U:{ }_{A} \mathrm{M} \rightarrow R_{R^{e}} \mathrm{M}$ is right adjoint to $A \otimes_{R^{e}}-:_{R^{e}} \mathrm{M} \rightarrow{ }_{A} \mathrm{M}$. Furthermore, Schauenburg's theorem states that bialgebroid structures on the $R^{e}$-ring $A$ are in one-to-one correspondance with monoidal 
structures on the category ${ }_{A} \mathrm{M}$ such that the forgetful functor $U$ is strict monoidal. At this point, the question arises whether a dual of this theorem holds for bicoalgebroids, namely: is there a one-to-one correspondance between bicoalgebroid structures on the coalgebra $\langle H, \bar{\Delta}, \bar{\varepsilon}\rangle$ and monoidal structures on the category ${ }^{H} \mathrm{M}$ such that $F:{ }^{H} \mathrm{M} \rightarrow{ }^{C} \mathrm{M}^{C}$ is strict monoidal? The next theorem gives the forward implication. We take up this question again in Section 4, and look at the reverse implication from a comonadic point of view.

Theorem 2.7. Let $H$ be a left bicoalgebroid over $C$. Then there is a monoidal structure on ${ }^{H} \mathrm{M}$ making the forgetful functor $F:{ }^{H} \mathrm{M} \rightarrow{ }^{C}{ }^{e} \mathrm{M} \simeq{ }^{C} \mathrm{M}^{C}$ strict monoidal. Identifying $H$-comodules with their underlying $C$-bicomodules, the monoidal product is $\square_{C}$, the cotensor product over $C$ and $C$ is the monoidal unit.

Proof. Assume there is a monoidal structure $\left\langle{ }^{H} \mathrm{M}, \odot, I\right\rangle$ on ${ }^{H} \mathrm{M}$ such that the forgetful functor is strict monoidal, meaning that we have a triple $\left\langle F, F^{2}, F^{0}\right\rangle$, where the maps $F^{M, N}: F(M \odot N) \rightarrow F(M) \square_{C} F(N)$ and $F^{0}: F(I) \rightarrow C$ are identities. This is tantamount to specifying

- an $H$-comodule structure on $C$,

$$
\delta_{C}: C \rightarrow H \square_{C^{e}} C, \text { and }
$$

- an $H$-comodule structure on the cotensor product of objects $M, N \in{ }^{C} \mathrm{M}^{C}$,

$$
\delta_{M \square N}: M \square_{C} N \rightarrow H \square_{C^{e}}\left(M \square_{C} N\right),
$$

natural in $M$ and $N$

The bicoalgebroid structure on $H$ allows us to construct such maps $\delta_{C}$ and $\delta_{M} \square N$.

The unit map $\eta: C \rightarrow H$ provides the desired $H$-comodule structure on $C$ :

$$
\delta_{C}=(H \otimes \alpha) \circ \Delta \circ \eta, \quad \delta_{C}(c)=\eta(c)_{(1)} \otimes \alpha\left(\eta(c)_{(2)}\right)
$$

This is indeed a coaction,

$$
\begin{aligned}
& \left(H \otimes \delta_{C}\right) \circ \delta_{C}(c)=\eta(c)_{(1)} \otimes \eta\left(\alpha\left(\eta\left(c_{(2)}\right)\right)\right)_{(1)} \otimes \alpha\left(\left(\eta\left(\alpha\left(\eta\left(c_{(2)}\right)\right)\right)_{(1)}\right)_{(2)}\right)= \\
& =\eta(c)_{(1)} \otimes \eta(c)_{(2)(1)} \otimes \alpha\left(\eta(c)_{(2)(2)}\right)=\eta(c)_{(1)(1)} \otimes \eta(c)_{(1)(2)} \otimes \alpha\left(\eta(c)_{(2)}\right)= \\
& =\left(\Delta_{H} \otimes C\right) \circ \delta_{C}(c),
\end{aligned}
$$

applying 2.5 in the second equality and coassociativity in the third.

For $M, N \in{ }^{H} \mathrm{M}$, define the coaction $\delta_{M} \square N: M \square_{C} N \rightarrow H \square_{C^{e}}\left(M \square_{C} N\right)$ as the composite map:

$$
\delta_{M \square N}: M \square_{C} \stackrel{\delta_{M} \square_{C} \delta_{N}}{\longrightarrow}\left(H \square_{C^{e}} M\right) \square_{C}\left(H \square_{C^{e}} N\right) \stackrel{\kappa}{\longrightarrow} H \square_{C^{e}}\left(M \square_{C} N\right)
$$

Implicit in this definition is the map

$$
\kappa:\left(H \square_{C^{e}} M\right) \square_{C}\left(H \square_{C^{e}} N\right) \rightarrow H \square_{C^{e}}\left(M \square_{C} N\right)
$$


which we define as the unique arrow in the following diagram

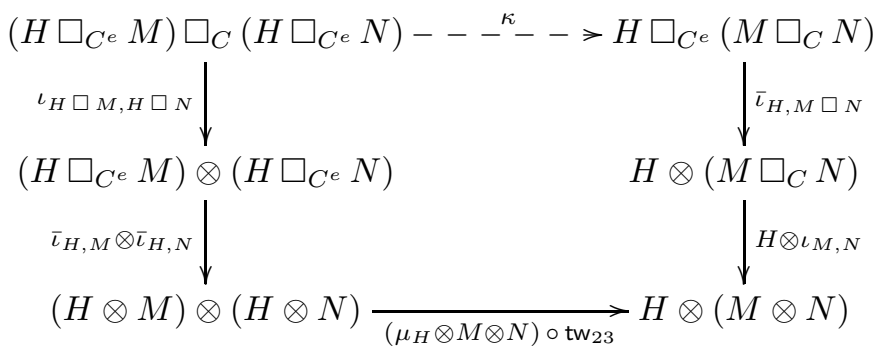

By the definition of the kernel maps $\iota_{M, N}: M \square_{C} N \rightarrow M \otimes N$ and $\bar{\iota}_{U, V}$ : $U \square_{C^{e}} V \rightarrow U \otimes V,(h \otimes m) \otimes\left(h^{\prime} \otimes n\right) \in\left(H \square_{C^{e}} M\right) \square_{C}\left(H \square_{C^{e}} N\right)$ if and only if the following identities hold:

$$
\begin{aligned}
\left(h_{(1)} \otimes \alpha\left(h_{(2)}\right) \otimes m\right) \otimes\left(h^{\prime} \otimes n\right) & =\left(h \otimes m_{[-1]} \otimes m_{[0]}\right) \otimes\left(h^{\prime} \otimes n\right) \\
\left(h_{(1)} \otimes \beta\left(h_{(2)}\right) \otimes m\right) \otimes\left(h^{\prime} \otimes n\right) & =\left(h \otimes m_{[1]} \otimes m_{[0]}\right) \otimes\left(h^{\prime} \otimes n\right) \\
(h \otimes m) \otimes\left(h_{(1)}^{\prime} \otimes \alpha\left(h_{(2)}^{\prime}\right) \otimes n\right) & =(h \otimes m) \otimes\left(h^{\prime} \otimes n_{[-1]} \otimes n_{[0]}\right) \\
(h \otimes m) \otimes\left(h_{(1)}^{\prime} \otimes \beta\left(h_{(2)}^{\prime}\right) \otimes n\right) & =(h \otimes m) \otimes\left(h^{\prime} \otimes n_{[1]} \otimes n_{[0]}\right)
\end{aligned}
$$

and

$$
h_{(2)} \otimes m \otimes \beta\left(h_{(1)}\right) \otimes h^{\prime} \otimes n=h \otimes m \otimes \alpha\left(h_{(1)}^{\prime}\right) \otimes h_{(2)}^{\prime} \otimes n
$$

The arrow $\kappa$ is defined by the universal property of the composite kernel map $\left(H \otimes \iota_{M, N}\right) \circ \bar{\iota}_{H, M \square N}$, provided

$$
\begin{array}{r}
\left(\mu_{H} \otimes M \otimes N\right) \circ \operatorname{tw}_{23} \circ\left(\bar{\iota}_{H, M} \otimes \bar{\iota}_{H, N}\right) \circ \iota_{H \square M, H \square N}\left((h \otimes m) \otimes\left(h^{\prime} \otimes n\right)\right)= \\
=\left(h h^{\prime}\right) \otimes(m \otimes n) \in H \square_{C^{e}}\left(M \square_{C} N\right)
\end{array}
$$

This leads to the following equations:

$$
\begin{aligned}
\left(h h^{\prime}\right) \otimes m_{[0]} \otimes m_{[1]} \otimes n & =\left(h h^{\prime}\right) \otimes m \otimes n_{[-1]} \otimes n_{[0]} \\
\left(h h^{\prime}\right)_{(1)} \otimes \alpha\left(\left(h h^{\prime}\right)_{(2)}\right) \otimes m \otimes n & =\left(h h^{\prime}\right) \otimes m_{[-1]} \otimes m_{[0]} \otimes n \\
\left(h h^{\prime}\right)_{(1)} \otimes \beta\left(\left(h h^{\prime}\right)_{(2)}\right) \otimes m \otimes n & =\left(h h^{\prime}\right) \otimes n_{[1]} \otimes m \otimes n_{[0]}
\end{aligned}
$$

Observe that by the multiplicativity of the coproduct and because $\left(H, \lambda_{L}, \rho_{L}\right)$ is a monoid in ${ }^{C} \mathrm{M}^{C}$, we have the following identities:

$$
\begin{aligned}
& \alpha\left(h h^{\prime}\right)=\alpha(h) \varepsilon\left(h^{\prime}\right) \\
& \beta\left(h h^{\prime}\right)=\varepsilon(h) \beta\left(h^{\prime}\right)
\end{aligned}
$$

To show 2.26, compute

$$
\alpha\left(h h^{\prime}\right)=\alpha\left(\left(h h^{\prime}\right)_{(1)}\right) \varepsilon\left(\left(h h^{\prime}\right)_{(2)}\right)=\alpha\left(h_{(1)}\right) \varepsilon\left(h_{(2)}\right) \varepsilon\left(h^{\prime}\right)=\alpha(h) \varepsilon\left(h^{\prime}\right),
$$

and analagously for 2.27. Note that 2.26 and 2.27 are dual to the relations $\Delta_{A}(t(r))=$ $1_{A} \otimes t(r)$ and $\Delta_{A}(s(r))=s(r) \otimes 1_{A}$, which hold for a left bialgebroid $A$ over $R$.

To prove 2.24, use 2.26 in the first equality and 2.18 in the second:

$$
\begin{aligned}
\left(h h^{\prime}\right)_{(1)} \otimes \alpha\left(\left(h h^{\prime}\right)_{(2)}\right) \otimes m \otimes n & =h_{(1)} h^{\prime} \otimes \alpha\left(h_{(2)}\right) \otimes m \otimes n= \\
& =\left(h h^{\prime}\right) \otimes m_{[-1]} \otimes m_{[0]} \otimes n
\end{aligned}
$$


Similarly, 2.25 is by proved by using 2.27 in the first equality and 2.20 in the second:

$$
\begin{aligned}
\left(h h^{\prime}\right)_{(1)} \otimes \beta\left(\left(h h^{\prime}\right)_{(2)}\right) \otimes m \otimes n & =h h_{(1)}^{\prime} \otimes \beta\left(h_{(2)}^{\prime}\right) \otimes m \otimes n= \\
& =\left(h h^{\prime}\right) \otimes n_{[1]} \otimes m \otimes n_{[0]},
\end{aligned}
$$

To prove 2.23, applying 2.19 and 2.20 to the left- and right hand sides, respectively, yields

$$
h_{(1)} h^{\prime} \otimes m \otimes \beta\left(h_{(2)}\right) \otimes n=h h_{(1)}^{\prime} \otimes m \otimes \alpha\left(h_{(2)}^{\prime}\right) \otimes n
$$

which holds precisely because multiplication satisfies the property 2.1

Let us check that $\delta_{M, N}: M \square_{C} N \rightarrow H \square_{C^{e}}\left(M \square_{C} N\right)$ is indeed a coaction. Expressed on elements, $\delta_{M, N}(m \otimes n)=m_{[-1]} n_{[-1]} \otimes m_{[0]} \otimes n_{[0]}$ (we think of the domain and range of $\delta_{M, N}$ as embedded into $M \otimes N$ and $H \otimes(M \otimes N)$, respectively).

$$
\begin{aligned}
\left(H \square \delta_{M, N}\right) \circ \delta_{M, N}(m \otimes n) & =m_{[-1]} n_{[-1]} \otimes m_{[0][-1]} n_{[0][-1]} \otimes m_{[0]} \otimes n_{[0]}= \\
& =m_{[-1](1)} n_{[-1](1)} \otimes m_{[-1](2)} n_{[-1](2)} \otimes m_{[0]} \otimes n_{[0]}= \\
& =\left(m_{[-1]} n_{[-1]}\right)_{(1)} \otimes\left(m_{[-1]} n_{[-1]}\right)_{(2)} \otimes m_{[0]} \otimes n_{[0]}= \\
& =(\Delta \otimes M) \circ \delta_{M, N}(m \otimes n)
\end{aligned}
$$

where we used the comultiplicativity of the multiplication on $H$ in the third equality.

For $\left\langle{ }^{H} \mathrm{M}, \square, C\right\rangle$ to be a monoidal category, we have still to define the natural isomorphisms $\alpha_{M, N, P}:(M \square N) \square P \rightarrow M \square(N \square P)$ (the associator), $\lambda_{M}$ : $C \square M \rightarrow M$ and $\rho_{N}: N \square C \rightarrow N$. Due to the strict monoidality of $F$, these maps may be defined as the lifting of the respective coherence morphisms of ${ }^{C} \mathrm{M}^{C}$ to ${ }^{H} \mathrm{M}^{H}$, provided they induce $H$-comodule maps. This, however, follows from the associativity and unit property of the multiplication and unit on $H$.

2.2. Modules over a bicoalgebroid. We proceed to define modules over a bicoalgebroid, especially for the purposes of Section 3.

Definition 2.8. A right module over a left bicoalgebroid $H$ (over $C$ ) is a pair $\langle X, \triangleleft\rangle$, where $X \in \mathrm{M}^{C}$ is a right $C$-comodule and the action is a right $C$-comodule map $\triangleleft: X \square_{C} H^{C} \rightarrow X^{C}$. Similarly, a left module is a pair $\langle Y, \triangleright\rangle$ with $Y \in{ }^{C} \mathrm{M}$ and $\triangleright:{ }^{C} H \square_{C} Y \rightarrow Y$ a left $C$-comodule map. $H$ is a $C$-bicomodule through the coactions $\lambda_{L}$ and $\rho_{L}$.

The module category of a bicoalgebroid is expected to be monoidal as well, coming with an embedding into ${ }^{C} \mathrm{M}^{C}$. The above definition doesn't seem to allow for this, but luckily, a dual of Prop. 1.1. of [1] holds:

Proposition 2.9. Let $\langle X, \triangleleft\rangle$ be a right module over the bicoalgebroid $H$. Then $X$ has a unique left $C$-comodule structure such that

(1) $X$ is a $C$-bicomodule

(2) the action is a $C$-bicomodule map

(3) $\triangleleft: X \square_{C} H \rightarrow X$ factorizes through $X \otimes H$

Proof. Note that the action being a right $C$-comodule map means

$$
(x \triangleleft h)_{[0]} \otimes(x \triangleleft h)_{[1]}=x \triangleleft h_{(2)} \otimes \beta\left(h_{(1)}\right)
$$

The left comodule structure in question will be denoted $\tau(x)=x_{[-1]} \otimes x_{[0]}$. In fact, $\tau$ is uniquely determined by demanding that the right $H$ action be also a left 
$C$-comodule map w.r.t $\tau$. Note that $X \square_{C} H$ is a left $C$-comodule through the left $C$-coaction $\lambda_{R}(h)=\beta\left(h_{(2)}\right) \otimes h_{(1)}$ on $H$, i.e. we impose:

$$
(x \triangleleft h)_{[-1]} \otimes(x \triangleleft h)_{[0]}=\beta\left(h_{(2)}\right) \otimes x \triangleleft h_{(1)}
$$

The identity $x=x_{[0]} \triangleleft \eta\left(x_{[1]}\right)$ and 2.29 yield an explicit formula for the left coaction $\tau$ :

$$
\begin{aligned}
x_{[-1]} \otimes x_{[0]} & =\left(x_{[0]} \triangleleft \eta\left(x_{[1]}\right)\right)_{[-1]} \otimes\left(x_{[0]} \triangleleft \eta\left(x_{[1]}\right)\right)_{[0]}= \\
& =\beta\left(\eta\left(x_{[1]}\right)_{(2)}\right) \otimes x_{[0]} \triangleleft \eta\left(x_{[1]}\right)_{(1)}
\end{aligned}
$$

This is indeed a coaction, i.e. $(C \otimes \tau) \circ \tau=\left(\Delta_{C} \otimes X\right) \circ \tau$. Inserting definitions, the

$$
\begin{aligned}
L H S=\beta\left(\eta\left(x_{[1]}\right)_{(2)}\right) & \otimes \beta\left\{\eta\left[\left(x_{\langle 0\rangle} \triangleleft \eta\left(x_{\langle 1\rangle}\right)_{(1)}\right)_{[1]}\right]_{(2)}\right\} \otimes \\
& \otimes\left(x_{\langle 0\rangle} \triangleleft \eta\left(x_{\langle 1\rangle}\right)_{(1)}\right)_{[0]} \triangleleft \eta\left[\left(x_{\langle 0\rangle} \triangleleft \eta\left(x_{\langle 1\rangle}\right)_{(1)}\right)_{[1]}\right]_{(1)}
\end{aligned}
$$

Using 2.28 , we find:

$$
\begin{aligned}
& L H S=\beta\left(\eta\left(x_{[1]}\right)_{(2)}\right) \otimes \beta\left\{\eta\left[\alpha\left(\eta\left(x_{[1]}\right)_{(1)(2)}\right)\right]_{(2)}\right\} \otimes \\
& \qquad\left(x_{[0]} \triangleleft \eta\left(x_{[0]}\right)_{(1)(1)}\right) \triangleleft \eta\left[\alpha\left(\eta\left(x_{[1]}\right)_{(1)(2)}\right)\right]_{(1)}
\end{aligned}
$$

which, by the bicoalgebroid axiom 2.5, is further equal:

$$
\begin{aligned}
L H S & =\beta\left(\eta\left(x_{[1]}\right)_{(2)}\right) \otimes \beta\left(\eta\left(x_{[1]}\right)_{(1)(2)}\right)_{(2)} \otimes x_{[0]} \triangleleft \eta\left(x_{[1]}\right)_{(1)(1)} \eta\left(x_{[1]}\right)_{(1)(2)(1)}= \\
& =\beta\left(\eta\left(x_{[1]}\right)_{(3)}\right) \otimes \beta\left(\eta\left(x_{[1]}\right)_{(2)}\right) \otimes x_{[0]} \triangleleft \eta\left(x_{[1]}\right)_{(1)}=\beta\left(\eta\left(x_{[1]}\right)_{(2)}\right)_{(1)} \otimes \\
& \otimes \beta\left(\eta\left(x_{[1]}\right)_{(2)}\right)_{(2)} \otimes x_{[0]} \triangleleft \eta\left(x_{[1]}\right)_{(1)}=R H S .
\end{aligned}
$$

In the first equality, we used comultiplicativity of the unit and coassociativity. In the second, the fact that $\beta$ is an anti-coalgebra map.

As for (1), the coaction $\tau$ makes $X$ a bicomodule. Using the definition of the left coaction, and that the $H$-action is a right $C$ comodule map:

$$
\begin{aligned}
x_{[-1]} & \otimes x_{[0][0]} \otimes x_{[0][1]}=\beta\left(\eta\left(x_{[1]}\right)_{(2)}\right) \otimes\left(x_{[0]} \triangleleft \eta\left(x_{[1]}\right)_{(1)}\right)_{[0]} \otimes \\
& \otimes\left(x_{[0]} \triangleleft \eta\left(x_{[1]}\right)_{(1)}\right)_{[1]}=\beta\left(\eta\left(x_{[1]}\right)_{(2)}\right) \otimes x_{[0]} \triangleleft \eta\left(x_{[1]}\right)_{(1)(2)} \otimes \beta\left(\eta\left(x_{[1]}\right)_{(1)(1)}\right)
\end{aligned}
$$

Using that $\eta: C^{C} \rightarrow H^{C}$ is a $C$-bicomodule map,

$$
\eta\left(c_{(1)}\right) \otimes c_{(2)}=\eta(c)_{(2)} \otimes \beta\left(\eta(c)_{(1)}\right)
$$

and the coassociativity of the coaction:

$$
\begin{aligned}
x_{[-1]} & \otimes x_{[0][0]} \otimes x_{[0][1]}=\beta\left(\eta\left(x_{[1]}\right)_{(2)(2)}\right) \otimes x_{[0]} \triangleleft \eta\left(x_{[1]}\right)_{(2)(1)} \otimes \beta\left(\eta\left(x_{[1]}\right)_{(1)}\right)= \\
& =\beta\left(\eta\left(x_{[1](1)}\right)_{(2)}\right) \otimes x_{[0]} \triangleleft \eta\left(x_{[1](1)}\right)_{(1)} \otimes x_{[1](2)}=\beta\left(\eta\left(x_{[0][1]}\right)_{(2)}\right) \otimes \\
& \otimes x_{[0][0]} \triangleleft \eta\left(x_{[0][1]}\right)_{(1)} \otimes x_{[1]}=x_{[0][-1]} \otimes x_{[0][0]} \otimes x_{[1]}
\end{aligned}
$$

(we apply 2.30 to $c=x_{[1]}$ in the second equality). The action will then (by construction) be a $C$-bicomodule map, proving (2). It remains to see that the action factorizes through the cocenter of $X \square_{C} H$, meaning:

$$
\left(x_{[0]} \triangleleft \eta\left(x_{[1]}\right)_{(1)}\right) \triangleleft h \otimes \beta\left(\eta\left(x_{[1]}\right)_{(2)}\right)=x_{[0]} \triangleleft h \otimes x_{[-1]}
$$

This is a simple consequence of 2.29 .

$$
L H S=\left(x_{[0]} \triangleleft \eta\left(x_{[1]}\right)\right)_{[0]} \triangleleft h \otimes\left(x_{[0]} \triangleleft \eta\left(x_{[1]}\right)\right)_{[-1]}=R H S .
$$




\section{THE SCALAR EXTENSION FOR BICOALGEBROIDS}

In [2], the authors introduced a construction that associates to a bialgebra $H$ and a braided commutative algebra $Q$ over $H$ a bialgebroid. In [1, it was shown that the construction generalizes to bialgebroids (in fact, even to Frobenius Hopfalgebroids) and has an interpretation as the noncommutative scalar extension of $H$ by $Q$.

Here we dualize this construction to bicoalgebroids, and give a few simple examples. We begin by defining the smash coproduct ([14, with a slight variation).

Definition 3.1. Let $H$ be a bicoalgebroid over $C$ and $D$ an $H$-comodule coalgebra. Then their smash coproduct $D \sharp H$ is a coalgebra, isomorphic to $D \square_{C} H$ as $C$ bicomodules and with the coalgebra structure:

$$
\begin{aligned}
\Delta(d \sharp h) & =d_{(1)} \sharp d_{(2)}{ }^{\langle-1\rangle} h_{(1)} \square_{D} d_{(2)}{ }^{\langle 0\rangle} \sharp h_{(2)} \\
\varepsilon(d \sharp h) & =\varepsilon(d) \varepsilon_{H}(h)
\end{aligned}
$$

That these maps define a coalgebra is easily verified. The category of $(D \sharp H)$ comodules may also be described as the internal $D$-comodules in ${ }^{H} \mathrm{M}$, i.e. ${ }^{D}\left({ }^{H} \mathrm{M}\right)=$ $D \sharp H \mathrm{M}$.

Indeed, assume $X \in{ }^{D}\left({ }^{H} \mathrm{M}\right)$. To every coaction $\delta_{D}: X \rightarrow D \square_{C} X$ in ${ }^{H} \mathrm{M}$, we can associate a coaction of $D \sharp H$, namely $\delta_{D \sharp H}=(D \otimes \delta) \circ \delta_{D}: X \rightarrow D \otimes X \rightarrow$ $D \otimes(H \otimes X), \delta_{D \sharp H}(x)=x_{[-1]} \otimes x_{[0]}{ }^{\langle-1\rangle} \otimes x_{[0]}{ }^{\langle 0\rangle}$. A straightforward calculation proves that $\left(\Delta_{D \sharp H} \otimes X\right) \circ \delta_{D \sharp H}=\left((D \sharp H) \otimes \delta_{D \sharp H}\right) \circ \delta_{D \sharp H}$, using that $\delta_{D \sharp H}$ is an $H$-comodule map. In the reverse direction, an $(D \sharp H)$-comodule is both an $H$-comodule and a $D$-comodule such that the $D$-coaction is an $H$-comodule map, which means precisely that it is an internal $D$-comodule in ${ }^{H} \mathrm{M}$.

3.1. Cocommutative coalgebras over bicoalgebroids. Keeping with the method of reversing arrows, we arrive at the following definition for Yetter-Drinfel'd modules over a bicoalgebroid.

Definition 3.2. Let $H$ be a (left-) bicoalgebroid over $C$. A Yetter-Drinfel'd module over $H$ is a triple $\langle Z, \triangleleft, \delta\rangle$ such that the $C$-bicomodule $Z$ is simultaneously a right $H$-module with $\triangleleft: Z \square_{C} H \rightarrow Z$ and a left $H$-comodule with $\delta: Z \rightarrow H \square_{C e} Z$ so that the action and coaction satisfy the compatibility condition

$$
d^{\langle-1\rangle} h_{[1]} \square_{C} d^{\langle 0\rangle} \triangleleft h_{[2]}=h_{[2]}\left(d \triangleleft h_{[1]}\right)^{\langle-1\rangle} \square_{C}\left(d \triangleleft h_{[1]}\right)^{\langle 0\rangle}
$$

The Yetter-Drinfel'd category, denoted ${ }^{H} \mathcal{Y} \mathcal{D}_{H}$ over $H$ has objects the YetterDrinfel'd modules over $H$ and arrows the $C$-bicomodule maps that are at the same time $H$-module maps and $H$-comodule maps.

The category ${ }^{H} \mathcal{Y} \mathcal{D}_{H}$ becomes monoidal if we define the monoidal product of two Yetter-Drinfel'd modules $Z, Z^{\prime}$ as $Z \square_{C} Z^{\prime}$ with action and coaction:

$$
\begin{gathered}
\left(z \square_{C} z^{\prime}\right) \triangleleft h=z \triangleleft h_{(2)} \square_{C} z^{\prime} \triangleleft h_{(1)} \\
\left(z \square_{C} z^{\prime}\right)_{\langle-1\rangle} \square_{C}\left(z \square_{C} z^{\prime}\right)_{\langle 0\rangle}=z_{\langle-1\rangle} z_{\langle-1\rangle}^{\prime} \square_{C} z_{\langle 0\rangle} \square_{C} z_{\langle 0\rangle}^{\prime}
\end{gathered}
$$

The monoidal unit is of course $C$, with $c \triangleleft h=c \varepsilon(h)$ and $c_{\langle-1\rangle} \otimes c_{\langle 0\rangle}=\eta\left(c_{(1)}\right) \otimes c_{(2)}$. Moreover, ${ }^{H} \mathcal{Y} \mathcal{D}_{H}$ is pre-braided with

$$
\tau_{Z, Z^{\prime}}: Z \square_{C} Z^{\prime} \rightarrow Z^{\prime} \square_{C} Z, \quad z \otimes z^{\prime} \mapsto z_{\langle 0\rangle}^{\prime} \square_{C} z \triangleleft z_{\langle-1\rangle}^{\prime}
$$


From experience with Hopf algebras, weak Hopf algebras and bialgebroids, it is reasonable to expect that the Yetter-Drinfel'd category over a bicoalgebroid is related to the (weak) center of the category of comodules. For the center construction, consult [12, 6] and [10]. The notion of weak center of a monoidal category seems to appear in [15, Definition 4.3 (see also [4, Section 1.3 and [1).

For bialgebroids, it is known that the Yetter-Drinfel'd category is equivalent to the monoidal weak center (see [15]). Unfortunately this doesn't seem to be true for bicoalgebroids in general. Nevertheless, the $\mathcal{Y} \mathcal{D}$ category over a bicoalgebroid still embeds into the monoidal weak center. Although the weak center construction is applicable to any monoidal category, we shall only recall the definition in the context of the comodule category over a bicoalgebroid.

For a bicoalgebroid $H$ over $C$, the (left) weak center $\overrightarrow{\mathcal{Z}}\left({ }^{H} \mathrm{M}\right)$ has objects $\langle Z, \theta\rangle$, where $Z \in{ }^{H} \mathrm{M}$ and $\theta$ is a natural transformation $\theta_{Y}: Z \square_{C} Y \rightarrow Y \square_{C} Z$ (between endofunctors on ${ }^{H} \mathrm{M}$ ) that satisfies

$$
\begin{aligned}
\theta_{X} \square_{C} Y & =\left(X \square_{C} \theta_{Y}\right) \circ\left(\theta_{X} \square_{C} Y\right) \\
\theta_{C} & =Z
\end{aligned}
$$

An arrow $\langle Z, \theta\rangle \rightarrow\left\langle Z^{\prime}, \theta^{\prime}\right\rangle$ is an $H$-comodule map $f: Z \rightarrow Z^{\prime}$, compatible with $\theta^{\prime}$ s in the sense:

$$
\left(Y \square_{C} f\right) \circ \theta_{Y}=\theta_{Y}^{\prime} \circ\left(f \square_{C} Y\right)
$$

for all $Y \in{ }^{H} \mathrm{M}$. The category $\overrightarrow{\mathcal{Z}}\left({ }^{H} \mathrm{M}\right)$ is monoidal and pre-braided with monoidal product

$$
\langle Z, \theta\rangle \square_{C}\left\langle Z^{\prime}, \theta^{\prime}\right\rangle=\left\langle Z \square_{C} Z^{\prime},\left(\theta_{-} \square_{C} Z^{\prime}\right) \circ\left(Z \square_{C} \theta^{\prime}{ }_{-}\right)\right\rangle
$$

and pre-braiding

$$
\vec{\beta}_{\langle Z, \theta\rangle,\left\langle Z^{\prime}, \theta^{\prime}\right\rangle}=\theta_{Z^{\prime}}
$$

It is easily shown that every Yetter-Drinfel'd module $\langle Z, \delta, \triangleleft\rangle$ has the structure of an object in $\overrightarrow{\mathcal{Z}}\left({ }^{H} \mathrm{M}\right)$. The map

$$
\begin{aligned}
\theta_{X}: Z \square_{C} X & \rightarrow X \square_{C} Z \\
z \otimes x & \mapsto x^{\langle 0\rangle} \otimes z \triangleleft x^{\langle-1\rangle}
\end{aligned}
$$

is natural in $X$, since the arrows of $\overrightarrow{\mathcal{Z}}\left({ }^{H} \mathrm{M}\right)$ are $H$-comodule maps, $\theta_{C}=Z$ is trivially satisfied and

$$
\theta_{X \square Y}(x \otimes y)=x^{\langle 0\rangle} \otimes y^{\langle 0\rangle} \otimes z \triangleleft\left(x^{\langle-1\rangle} y^{\langle-1\rangle}\right)
$$

equals

$$
\begin{aligned}
\left(X \square \theta_{Y}\right) \circ\left(\theta_{X} \square Y\right)(x \otimes y) & =\left(X \square \theta_{Y}\right)\left(x^{\langle 0\rangle} \otimes z \triangleleft x^{\langle-1\rangle} \otimes y\right)= \\
& =x^{\langle 0\rangle} \otimes y^{\langle 0\rangle} \otimes\left(z \triangleleft x^{\langle-1\rangle}\right) \triangleleft y^{\langle-1\rangle} .
\end{aligned}
$$

As for the reverse direction, we can associate to every object $\langle Z, \theta\rangle$ of $\overrightarrow{\mathcal{Z}}\left({ }^{H} \mathrm{M}\right)$ a right action of $H$ as follows:

$$
\begin{aligned}
& \triangleleft: Z \square_{C} H \rightarrow Z \\
& z \otimes h \mapsto\left(\varepsilon_{H} \otimes Z\right) \circ \theta_{H}(z \otimes h)
\end{aligned}
$$


It is easily checked that this is indeed a right $H$-action, and is the candidate to make $Z$ a Yetter-Drinfel'd module. If $\theta$ enjoys the property $\theta_{X}(z \otimes x)=x^{\langle 0\rangle} \otimes$ $\theta_{H}\left(z \otimes x^{\langle-1\rangle}\right)$ for all objects $X \in{ }^{H} \mathrm{M}$, then $\langle Z, \delta, \triangleleft\rangle$ becomes a Yetter-Drinfel'd module and, moreover, ${ }^{H} \mathcal{Y} \mathcal{D}_{H}$ and $\overrightarrow{\mathcal{Z}}\left({ }^{H} \mathrm{M}\right)$ are isomorphic categories. This would mean that the natural map $\theta$ can be expressed with it's component $\theta_{H}$. This is indeed possible for bialgebroids, since any bialgebroid is a generator in the category of modules over itself, and natural transformations are determined by their value on the generator.

Remark 3.3. We mention, for the sake of completeness, the right weak center $\overleftarrow{\mathcal{Z}}\left({ }^{H} \mathrm{M}\right)$, defined as the category of pairs $\langle Z, \bar{\theta}\rangle$, where $Z \in{ }^{H} \mathrm{M}$ and $\bar{\theta}$ is a natural transformation $\theta_{Y}: Y \square_{C} Z \rightarrow Z \square_{C} Y$, satisfying

$$
\begin{aligned}
\bar{\theta}_{X} \square_{C} Y & =\left(\bar{\theta}_{X} \square_{C} Y\right) \circ\left(X \square_{C} \bar{\theta}_{Y}\right) \\
\theta_{C} & =Z
\end{aligned}
$$

The category $\overleftarrow{\mathcal{Z}}\left({ }^{H} \mathrm{M}\right)$ has the monoidal structure

$$
\langle Z, \bar{\theta}\rangle \square_{C}\left\langle Z^{\prime}, \bar{\theta}^{\prime}\right\rangle=\left\langle Z \square_{C} Z^{\prime},\left(Z \square_{C} \bar{\theta}_{-}^{\prime}\right) \circ\left(\bar{\theta}_{-} \square_{C} Z^{\prime}\right)\right\rangle
$$

and pre-braiding

$$
\overleftarrow{\beta}_{\langle Z, \bar{\theta}\rangle,\left\langle Z^{\prime}, \bar{\theta}^{\prime}\right\rangle}=\bar{\theta}_{Z}^{\prime}
$$

It is straightforward to prove that the one-sided Yetter-Drinfel'd category ${ }_{H}^{H} \mathcal{Y D}$ is embedded into $\overleftarrow{\mathcal{Z}}\left({ }^{H} \mathrm{M}\right)$. The objects of ${ }_{H}^{H} \mathcal{Y} \mathcal{D}$ are triples $\langle Z, \delta, \triangleright\rangle, C$-bicomodules which are simultaneously $H$-modules and $H$-comodules, satifying the compatibilty condition

$$
h_{(1)} z^{\langle-1\rangle} \square_{C} h_{(2)} \triangleright z^{\langle 0\rangle}=\left(h_{(1)} \triangleright z\right)^{\langle-1\rangle} h_{(2)} \square_{C}\left(h_{(1)} \triangleright z\right)^{\langle 0\rangle}
$$

${ }_{H}^{H} \mathcal{Y D}$ is a pre-braided monoidal category with the pre-braiding

$$
\kappa_{Z^{\prime}, Z}\left(z^{\prime} \otimes z\right)=z^{\prime\langle-1\rangle} \triangleright z \otimes z^{\langle 0\rangle}
$$

Now, a braided cocommutative coalgebra (hereinafter abbreviated BCC) over $H$ is defined as a cocommutative comonoid in ${ }^{H} \mathcal{Y} \mathcal{D}_{H}$. Spelled out in detail, we have the

Definition 3.4. A BCC over $H$ is a coalgebra $D$, equipped with a coalgebra map $\varepsilon: D \rightarrow C$ and the structure of a Yetter-Drinfel'd module $\langle D, \triangleleft, \delta\rangle \in{ }^{H} \mathcal{Y} \mathcal{D}_{H}$ so that the left/right $C$-comodule structures on $D$ are given by $\varepsilon\left(d_{(1)}\right) \otimes d_{(2)}$ and $d_{(1)} \otimes \varepsilon\left(d_{(2)}\right)$, respectively and the relations stating that $D$ is an $H$-module and $H$-comodule coalgebra:

$$
\begin{aligned}
& (d \triangleleft h)_{(1)} \otimes(d \triangleleft h)_{(2)}=d_{(1)} \triangleleft h_{(1)} \otimes d_{(2)} \triangleleft h_{(2)} \\
& \varepsilon(d \triangleleft h)=\varepsilon(d) \varepsilon_{H}(h) \\
& d_{(1)}{ }^{\langle-1\rangle} d_{(2)}{ }^{\langle-1\rangle} \otimes d_{(1)}{ }^{\langle 0\rangle} \otimes d_{(2)}{ }^{\langle 0\rangle}=d^{\langle-1\rangle} \otimes d^{\langle 0\rangle}{ }_{(1)} \otimes d^{\langle 0\rangle}{ }_{(2)} \\
& d^{\langle-1\rangle} \otimes \varepsilon\left(d^{\langle 0\rangle}\right)=\eta\left(\varepsilon(d)_{(1)}\right) \otimes \varepsilon(d)_{(2)}
\end{aligned}
$$

and braided cocommutativity:

$$
d_{(1)} \otimes d_{(2)}=d_{(2)}\langle 0\rangle \otimes d_{(1)} \triangleleft d_{(2)}\langle-1\rangle
$$


We have the following functorial characterization of BCC's, entirely analogous to Prop 4.7. of [1]:

Lemma 3.5. If $D$ is a $B C C$ in ${ }^{H} \mathcal{Y} \mathcal{D}_{H}$, then the functor $D \square_{C-}:{ }^{H} \mathrm{M} \rightarrow{ }^{D}\left({ }^{H} \mathrm{M}\right)=$ $D \sharp H \mathrm{M}$ is strong monoidal

Proof. Denote the opmonoidal structure $\left\langle D \square_{C-}, D^{2}, D^{0}\right\rangle$. The natural transformation

$$
\begin{aligned}
\left(D^{2}\right)_{X, Y}: D \square_{C}\left(X \square_{C} Y\right) \rightarrow\left(D \square_{C} X\right) \square_{D}\left(D \square_{C} Y\right) \\
d \otimes x \otimes y \mapsto\left(d_{(1)} \otimes x_{\langle 0\rangle}\right) \otimes\left(d_{(2)} \triangleleft x_{\langle-1\rangle} \otimes y\right)
\end{aligned}
$$

has the inverse $(d \otimes x) \otimes\left(d^{\prime} \otimes y\right) \mapsto d \varepsilon_{C}\left(\varepsilon\left(d^{\prime}\right)\right) \otimes x \otimes y$. Furthermore, $D^{0}: D \square_{C} C \rightarrow$ $D$ is obviously an isomorphism.

It is perhaps not altogether surprising that we have the following dualization of Theorem 4.6. of [1]

Theorem 3.6. Let $\langle H, \Delta, \varepsilon ; \mu, \eta ; \alpha, \beta ; C\rangle$ be a (left-) bicoalgebroid over $C$ and $D$ a $B C C$ over $H$, then $\langle D \sharp H, \tilde{\Delta}, \tilde{\varepsilon} ; \tilde{\mu}, \tilde{\eta} ; \tilde{\alpha}, \tilde{\beta} ; D\rangle$ is a (left-) bicoalgebroid over $D$, with the following structure maps:

$$
\begin{aligned}
\tilde{\Delta}(d \sharp h) & =d_{(1)} \sharp d_{(2)}{ }^{\langle-1\rangle} h_{(1)} \square_{D} d_{(2)}{ }^{\langle 0\rangle} \sharp h_{(2)} \\
\tilde{\varepsilon}(d \sharp h) & =\varepsilon_{C}(\varepsilon(d)) \varepsilon_{H}(h) \\
\tilde{\mu}\left(d \sharp h \square_{D} d^{\prime} \sharp h^{\prime}\right) & =d \varepsilon_{C}\left(\varepsilon\left(d^{\prime}\right)\right) \sharp h h^{\prime} \\
\tilde{\eta}(d) & =d_{(1)} \sharp \eta\left(\varepsilon\left(d_{(2)}\right)\right) \\
\tilde{\alpha}(d \sharp h) & =d \varepsilon_{H}(h), \quad \tilde{\beta}(d \sharp h)=d \triangleleft h
\end{aligned}
$$

Proof. First, we check that $\tilde{\alpha}(\tilde{\beta})$ is a coalgebra (anti-coalgebra) map, respectively. Inserting the definitions, a trivial calculation shows

$$
\alpha\left((d \sharp h)_{(1)}\right) \otimes \alpha\left((d \sharp h)_{(2)}\right)=d_{(1)} \otimes d_{(2)} \varepsilon(h)=(\alpha(d \sharp h))_{(1)} \otimes(\alpha(d \sharp h))_{(2)}
$$

As required, $\beta$ is an anti-coalgebra map:

$$
\begin{aligned}
& \beta\left((d \sharp h)_{(2)}\right) \otimes \beta\left((d \sharp h)_{(1)}\right)=\beta\left(d_{(2)}{ }^{\langle 0\rangle} \sharp h_{(2)}\right) \otimes \beta\left(d_{(1)} \sharp d_{(2)}{ }^{\langle-1\rangle} h_{(1)}\right)= \\
& =d_{(2)}\langle 0\rangle \triangleleft h_{(2)} \otimes d_{(1)} \triangleleft d_{(2)}\langle-1\rangle h_{(1)}=d_{(1)} \triangleleft h_{(2)} \otimes d_{(2)} \triangleleft h_{(1)}=(d \triangleleft h)_{(1)} \otimes(d \triangleleft h)_{(2)}= \\
& =(\beta(d \sharp h))_{(1)} \otimes(\beta(d \sharp h))_{(2)} \text {. }
\end{aligned}
$$

where we have used 3.22 in the third equality, and the fact that $D$ is an $H_{c o p}{ }^{-}$ coalgebra in the fourth.

To prove that $\tilde{\mu}:(D \sharp H) \square_{D}(D \sharp H) \rightarrow D \sharp H$ factorizes through $(D \sharp H) \otimes$ $(D \sharp H)$, we calculate the $D$-comodule structure of $D \sharp H$ :

$$
\begin{aligned}
& \tilde{\lambda}_{L}: d \sharp h \mapsto \alpha\left((d \sharp h)_{(1)}\right) \otimes(d \sharp h)_{(2)}=\alpha\left(d_{(1)} \sharp d_{(2)}{ }^{\langle-1\rangle} h_{(1)}\right) \otimes d_{(2)}{ }^{\langle 0\rangle} \sharp h_{(2)}= \\
& =d_{(1)} \otimes d_{(2)} \sharp h \\
& \tilde{\rho}_{L}: d \sharp h \mapsto(d \sharp h)_{(2)} \otimes \beta\left((d \sharp h)_{(2)}\right)=d_{(2)}{ }^{\langle 0\rangle} \sharp h_{(2)} \otimes d_{(1)} \triangleleft\left(d_{(2)}{ }^{\langle-1\rangle} h_{(1)}\right)= \\
& =d_{(1)} \sharp h_{(2)} \otimes d_{(2)} \triangleleft h_{(1)},
\end{aligned}
$$


using (3.22) in the last step. The definition of the cotensor product over $D$ then reads: $(d \otimes h) \otimes\left(d^{\prime} \otimes h^{\prime}\right) \in(D \sharp H) \square_{D}(D \sharp H)$ iff

$$
(d \sharp h)_{(2)} \otimes \beta\left((d \sharp h)_{(1)}\right) \otimes\left(d^{\prime} \sharp h^{\prime}\right)=(d \sharp h) \otimes \alpha\left(\left(d^{\prime} \sharp h^{\prime}\right)_{(1)}\right) \otimes\left(d^{\prime} \sharp h^{\prime}\right)_{(2)}
$$

or, using (3.22):

$$
d_{(1)} \sharp h_{(2)} \otimes d_{(2)} \triangleleft h_{(1)} \otimes d^{\prime} \sharp h^{\prime}=d \sharp h \otimes d_{(1)}^{\prime} \otimes d_{(2)}^{\prime} \sharp h^{\prime}
$$

We now prove $(d \sharp h)\left(d^{\prime} \sharp h^{\prime}\right)_{(1)} \otimes \alpha\left(\left(d^{\prime} \sharp h^{\prime}\right)_{(2)}\right)=(d \sharp h)_{(1)}\left(d^{\prime} \sharp h^{\prime}\right) \otimes \beta\left((d \sharp h)_{(2)}\right)$. Inserting definitions, and using the Yetter-Drinfel'd condition (3.3) we find:

$$
\begin{aligned}
R H S & =d_{(1)} \varepsilon_{C}\left(\varepsilon\left(d^{\prime}\right)\right) \sharp d_{(2)}\langle-1\rangle \\
& =d_{(1)} h_{(1)} \varepsilon_{C}\left(\varepsilon\left(d^{\prime}\right)\right) \sharp h_{(2)}\left(d_{(2)} \triangleleft h_{(1)}\right)^{\langle-1\rangle} h^{\prime} \otimes h_{(2)}= \\
& \left(d_{(2)} \triangleleft h_{(1)}\right)^{\langle 0\rangle},
\end{aligned}
$$

using the Yetter-Drinfel'd condition (eq. 3.3.3). Applying (3.28), we arrive at

$$
R H S=d \varepsilon_{C}\left(\varepsilon\left(d_{(2)}^{\prime}\right)\right) \sharp h d^{\prime}{ }_{(1)}{ }^{\langle-1\rangle} h^{\prime} \otimes d^{\prime}{ }_{(1)}{ }^{\langle 0\rangle}=d \sharp h d^{\prime\langle-1\rangle} h^{\prime} \otimes d^{\prime\langle 0\rangle}
$$

A quick calculation shows that the

$$
L H S=d \varepsilon_{C}\left(\varepsilon\left(d^{\prime}{ }_{(1)}\right)\right) \sharp h d^{\prime}{ }_{(2)}{ }^{\langle-1\rangle} h^{\prime} \otimes d^{\prime}{ }_{(2)}{ }^{\langle 0\rangle}=d \sharp h d^{\prime\langle-1\rangle} h^{\prime} \otimes d^{\prime\langle 0\rangle},
$$

as claimed.

Comultiplicativity of the product (which makes sense due to our above assertion) means

$$
(\tilde{\Delta} \circ \tilde{\mu})\left[(d \sharp h) \square_{D}\left(d^{\prime} \sharp h^{\prime}\right)\right]=\left(\tilde{\mu} \square_{D} \tilde{\mu}\right) \circ \tau_{23} \circ\left(\tilde{\Delta} \square_{D} \tilde{\Delta}\right)\left[(d \sharp h) \square_{D}\left(d^{\prime} \sharp h^{\prime}\right)\right]
$$

inserting our definitions, we have:

$$
L H S=\tilde{\Delta}\left(d \varepsilon_{C} \varepsilon\left(d^{\prime}\right) \sharp h h^{\prime}\right)=d_{(1)} \varepsilon_{C} \varepsilon\left(d^{\prime}\right) \sharp d_{(2)}{ }^{\langle-1\rangle}\left(h h^{\prime}\right)_{(1)} \square_{D} d_{(2)}{ }^{\langle 0\rangle} \sharp\left(h h^{\prime}\right)_{(2)},
$$

on the other hand, the

$$
\begin{aligned}
& R H S=\left(d_{(1)} \sharp d_{(2)}{ }^{\langle-1\rangle} h_{(1)}\right)\left(d_{(1)}^{\prime} \sharp d_{(2)}^{\prime}{ }^{\langle-1\rangle} h_{(1)}^{\prime}\right) \square_{D}\left(d_{(2)}{ }^{\langle 0\rangle} \sharp h_{(2)}\right)\left(d_{(2)}^{\prime}\langle 0\rangle \sharp h_{(2)}^{\prime}\right)= \\
& =d_{(1)} \varepsilon_{C} \varepsilon\left(d_{(1)}^{\prime}\right) \sharp d_{(2)}{ }^{\langle-1\rangle} h_{(1)} d_{(2)}^{\prime}{ }^{\langle-1\rangle} h_{(1)}^{\prime} \square_{D} d_{(2)}{ }^{\langle 0\rangle} \varepsilon_{C} \varepsilon\left(d_{(2)}^{\prime}{ }^{\langle 0\rangle}\right) \sharp h_{(2)} h_{(2)}^{\prime}= \\
& =d_{(1)} \varepsilon_{C}\left(\varepsilon\left(d_{(1)}^{\prime}\right)\right) \sharp d_{(2)}{ }^{\langle-1\rangle} h_{(1)} \eta\left(\varepsilon\left(d_{(2)}^{\prime}\right)\right) h_{(1)}^{\prime} \square_{D} d_{(2)}{ }^{\langle 0\rangle} \varepsilon_{C}\left(\varepsilon\left(d_{(3)}^{\prime}\right)\right) \sharp\left(h h^{\prime}\right)_{(2)},
\end{aligned}
$$

where we made use of 3.21 and coassociativity in the third equality. Now, $d_{(1)}^{\prime} \otimes$ $\varepsilon\left(d_{(2)}^{\prime}\right) \otimes h^{\prime}=d^{\prime} \otimes \alpha\left(h_{(1)}^{\prime}\right) \otimes h_{(2)}^{\prime}$, because $d \sharp h \in D \square_{C} H$. From this, and the unit property of $\eta$, the statement follows.

The product is counital:

$$
\tilde{\varepsilon}(d \sharp h) \tilde{\varepsilon}\left(d^{\prime} \sharp h^{\prime}\right)=\varepsilon_{C}(\varepsilon(d)) \varepsilon_{C}\left(\varepsilon\left(d^{\prime}\right)\right) \varepsilon_{H}\left(h h^{\prime}\right)=\tilde{\varepsilon}\left(d \varepsilon_{C}(\varepsilon(d)) \sharp h h^{\prime}\right)
$$

The unit map $\tilde{\eta}$ is indeed a unit for $\tilde{\mu}$. The first unit property reads:

$$
\begin{aligned}
& \tilde{\mu} \circ(\tilde{\eta} \square D \sharp H) \circ \tilde{\lambda}_{L}(d \sharp h)=\left(d_{(1)(1)} \sharp \eta\left(\varepsilon\left(d_{(1)(2)}\right)\right)\right)\left(d_{(2)} \sharp h\right)= \\
& =d_{(1)} \varepsilon_{C} \varepsilon\left(d_{(3)}\right) \sharp \eta\left(\varepsilon\left(d_{(2)}\right)\right) h=d \sharp h,
\end{aligned}
$$

using $d \sharp h \in D \square_{C} H$ and the unit axiom (for $H$ ) in the last equality. The second,

$$
\begin{aligned}
& \tilde{\mu} \circ(D \sharp H \square \tilde{\eta}) \circ \tilde{\rho}_{L}(d \sharp h)=\left(d_{(1)} \sharp h_{(2)}\right)\left(\left(d_{(2)} \triangleleft h_{(1)}\right)_{(1)} \sharp \eta\left(\left(d_{(2)} \triangleleft h_{(1)}\right)_{(2)}\right)\right)= \\
& =d_{(1)} \varepsilon_{C} \varepsilon\left(d_{(2)} \triangleleft h_{(1)}\right) \sharp h_{(2)} \eta\left(\varepsilon\left(d_{(2)} \triangleleft h_{(1)}\right)_{(2)}\right)=d_{(1)} \sharp \eta\left(\varepsilon\left(d_{(2)}\right)\right) h=d \sharp h
\end{aligned}
$$

is proved using that $D$ is an $H_{c o p}$-algebra in the third equality, and $d \sharp h \in D \square_{C} H$ in the last. As a coalgebra, $D \sharp H$ is the smash coproduct. The algebra structure of $\langle D \sharp H, \tilde{\mu}, \tilde{\eta}\rangle$ and the remaining axioms are easily verified. 
Example 3.7. The action groupoid

In the category Set, there is a unique comultiplication, namely the diagonal coproduct: $x \in X, \Delta_{X}(x)=x \times x$. The counit is just a constant map to a (the) one-element set 1 , hence $\varepsilon_{X}(x)=*$ for all $x \in X$, where $*$ is the unique element of 1 . The coaction of a group $G$ on $X$ is completely specified by an arbitrary function $\varphi: X \rightarrow G$, via $\delta_{\varphi}(x)=x_{\langle-1\rangle} \times x_{\langle 0\rangle}=\varphi(x) \times x$. Now, consider a $G$-Set $\langle X, \triangleleft\rangle$, carrying a right action of $G$. Choosing a $G$-coaction $\delta_{\varphi}$, the Yetter-Drinfel'd compatibility condition takes the form

$$
\varphi(x) g \times x \triangleleft g=g \varphi(x \triangleleft g) \times x \triangleleft g
$$

so $\left\langle X, \delta_{\varphi}, \triangleleft\right\rangle$ is a $\mathcal{Y} \mathcal{D}$-module in ${ }^{G} \mathcal{Y} \mathcal{D}_{G}$ if and only if $g^{-1} \varphi(x) g=\varphi(x \triangleleft g)$. Moreover, $X$ is a BCC if $x \times x=x \times x \triangleleft \varphi(x)$, i.e. iff

$$
x \triangleleft \varphi(x)=x
$$

3.32 implies that the value of $\varphi$ at a point $x$ must lie in the stabilizer subgroup $G^{x}$ of the point $x$, and from 3.31 we conclude that it suffices to define $\varphi$ for a single representative, say $x_{0}$ of each $G$-orbit. Then, if $x_{0} \in G^{x_{0}}, \varphi(x)=\varphi\left(x_{0} \triangleleft g\right)=$ $g^{-1} \varphi\left(x_{0}\right) g \in G^{x}$.

Choosing a trivial coaction $\varphi(x) \equiv e$, the scalar extension of $G$ by $X$ is nothing but the action groupoid. Indeed, $\tilde{\alpha}(x \sharp g)=x$ and $\tilde{\beta}(x \sharp g)=x \triangleleft g$, so $(X \sharp G) \square X(X \sharp G)$ is the set of composable pairs in the action groupoid and the multiplication $\tilde{\mu}$ is the composition of arrows in the action groupoid.

The phenomenon behind this example is that in Set, the fibered product of two parallel maps $\alpha, \beta: X \rightarrow Y$, defined by the pullback

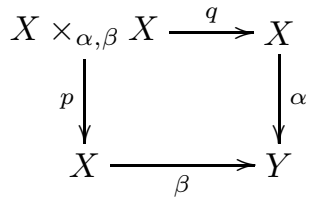

is equivalent to the equalizer

$$
X \times_{\alpha, \beta} X \rightarrow X \times X \underset{\rho \times X}{\stackrel{X \times \lambda}{\longrightarrow}} X \times Y \times X
$$

where $\lambda$ and $\rho$ are the 'coactions' $\lambda=(\alpha \times X) \circ \Delta_{\text {diag }}$ and $\rho=(X \times \beta) \circ \Delta_{\text {diag }}$. It is in this sense that a groupoid may be regarded as a classical ancestor of a bicoalgebroid.

Example 3.8. The regular $B C C$ for $H$ a Hopf algebra

$k$-Hopf algebras (and bialgebras) are examples both of bialgebroids and bicoalgebroids. It is not immaterial, however whether we consider the Yetter-Drinfel'd category ${ }^{H} \mathcal{Y} \mathcal{D}_{H}$ as embedded in $\overrightarrow{\mathcal{Z}}\left(\mathrm{M}_{H}\right)$ (the 'bialgebroid view', see [1]), or in $\overrightarrow{\mathcal{Z}}\left({ }^{H} \mathrm{M}\right)$ (the 'bicoalgebroid view'). Namely, the braiding is different in the two cases, $\overrightarrow{\mathcal{Z}}\left(\mathrm{M}_{H}\right)$ is pre-braided with $\vec{\beta}_{Z, Z^{\prime}}=z^{\prime} \triangleleft z^{\langle-1\rangle} \otimes z^{\langle 0\rangle}$ and $\overrightarrow{\mathcal{Z}}\left({ }^{H} \mathrm{M}\right)$ is prebraided with $\vec{\gamma}_{Z, Z^{\prime}}={z^{\prime}}^{\langle 0\rangle} \otimes z \triangleleft z^{\langle\langle-1\rangle}$.

A $k$-Hopf algebra $H$, with invertible antipode is a Yetter-Drinfel'd module $\left\langle H, A d_{R}, \Delta\right\rangle$ in $\overrightarrow{\mathcal{Z}}\left(\mathrm{M}_{H}\right)$ (the regular module) via the coproduct, considered as 
left $H$-coaction and the right adjoint action, $A d_{R}: H \otimes H \rightarrow H, \quad h \otimes h^{\prime} \mapsto$ $S^{-1}\left(h_{(2)}^{\prime}\right) h h_{(1)}^{\prime}$. Furthermore, $H^{o p}$ is a BCA in $\overrightarrow{\mathcal{Z}}\left(\mathrm{M}_{H}\right)$, that is $\mu_{o p} \circ \beta\left(h \otimes h^{\prime}\right)=$ $\mu_{o p}\left(h \otimes h^{\prime}\right)$. Indeed, $h^{\prime} h=h^{\prime} \triangleleft h_{(1)} \otimes h_{(2)}=S\left(h_{(1)(2)}\right) h^{\prime} h_{(1)(1)} \otimes h_{(2)}$.

Dually, a $k$-Hopf algebra $H$ is a Yetter-Drinfel'd module $\left\langle H, \mu, \tilde{A} d_{L}\right\rangle$ in $\overrightarrow{\mathcal{Z}}\left({ }^{H} \mathrm{M}\right)$ (the regular module) via the multiplication considered as a right action, and the left adjoint coaction,

$$
\begin{aligned}
\tilde{A} d_{L}: H & \rightarrow H \otimes H \\
h & \mapsto S^{-1}\left(h_{(3)}\right) h_{(1)} \otimes h_{(2)}
\end{aligned}
$$

Yetter-Drinfel'd compatibility is easily checked:

$$
\begin{aligned}
& h_{(2)}^{\prime}\left(h h_{(1)}^{\prime}\right)^{\langle-1\rangle} \otimes\left(h h_{(1)}^{\prime}\right)^{\langle 0\rangle}=h_{(2)}^{\prime} S^{-1}\left(\left(h h_{(1)}^{\prime}\right)_{(3)}\right)\left(h h_{(1)}^{\prime}\right) \otimes\left(h h_{(2)}^{\prime}\right)= \\
& =h_{(2)}^{\prime} S^{-1}\left(h_{(1)(3)}^{\prime}\right) S^{-1}\left(h_{(3)}\right) h_{(1)(1)}^{\prime} h_{(1)} \otimes h_{(2)} h_{(1)(2)}^{\prime}=S^{-1}\left(h_{(3)}\right) h_{(1)} h_{(1)}^{\prime} \otimes h_{(2)} h_{(2)}^{\prime}= \\
& =h^{\langle-1\rangle} h_{(1)}^{\prime} \otimes h^{\langle 0\rangle} \triangleleft h_{(2)}^{\prime}
\end{aligned}
$$

As one might expect from the previous example, $H_{\text {cop }}$ is a BCC in $\overrightarrow{\mathcal{Z}}\left({ }^{H} \mathrm{M}\right)$,

$$
\vec{\beta} \circ \Delta_{c o p}(h)=\vec{\beta}\left(h_{(2)} \otimes h_{(1)}\right)=h_{(1)(2)} \otimes h_{(2)} S^{-1}\left(h_{(1)(3)}\right) h_{(1)(1)}=h_{(2)} \otimes h_{(1)}
$$

To construct an example which does not require the invertibility of the antipode, consider ${ }_{H}^{H} \mathcal{Y D}$ as being in the right weak center $\overleftarrow{\mathcal{Z}}\left({ }^{H} \mathrm{M}\right)$. The Yetter-Drinfel'd condition takes the form

$$
h_{(1)} z^{\langle-1\rangle} \otimes h_{(2)} \triangleright z^{\langle 0\rangle}=\left(h_{(1)} \triangleright z\right)^{\langle-1\rangle} h_{(2)} \otimes\left(h_{(1)} \triangleright z\right)^{\langle 0\rangle},
$$

and the pre-braiding is $\overleftarrow{\beta}_{Z^{\prime}, Z}:{z^{\prime}}^{\langle-1\rangle} \triangleright z \otimes z^{\prime\langle 0\rangle}$. We find that for an arbitrary Hopf algebra, $\left\langle H, A d_{L}, \mu_{H}\right\rangle$ is a BCC in ${ }_{H}^{H} \mathcal{Y D}$, where

$$
\begin{aligned}
A d_{L}: H & \rightarrow H \otimes H \\
h & \mapsto h_{(1)} S\left(h_{(3)}\right) \otimes h_{(2)},
\end{aligned}
$$

and $\overleftarrow{\beta}_{H, H} \circ \Delta(h)=h_{(1)(1)} S\left(h_{(1)(3)}\right) h_{(2)} \otimes h_{(1)(2)}=h_{(1)} \otimes h_{(2)}$

\section{The scalar EXTEnsion as a COMOnAD}

In this section, we give a (co-)monadic characterization of bicoalgebroids which can be seen as dual to the results obtained for bialgebroids in [17. We also give a categorical description of the bialgebroid and bicoalgebroid scalar extensions in terms of bimonads, and bicomonads, respectively.

Recall that for a bicoalgebroid $H$, the forgetful functor $F:{ }^{H} \mathrm{M} \rightarrow{ }^{e}{ }^{e} \mathrm{M}$ is strong monoidal, and is left adjoint to the induction functor $I={ }^{H} H \square_{C^{e}}:{ }^{C}{ }^{e} \mathrm{M} \rightarrow{ }^{H} \mathrm{M}$. By the standard Eilenberg-Moore construction (see [11), the adjunction $F \dashv I$ gives rise to a monad $\mathbb{T}=\langle T, \mu, \eta\rangle$ on the category ${ }^{H} \mathrm{M}$ with underlying endofunctor $T=I F:{ }^{H} \mathrm{M} \rightarrow{ }^{H} \mathrm{M}$ ( monad multiplication is $\mu=I \varepsilon F: T T \rightarrow T$, monad unit $\eta:{ }^{H} \mathrm{M} \rightarrow T$ is the unit of the adjunction) and a comonad $\mathbb{G}=\langle G, \Delta, \varepsilon\rangle$ on the category ${ }^{C} \mathrm{M}$ with underlying endofunctor $G=F I:{ }^{e} \mathrm{M} \rightarrow{ }^{C} \mathrm{M}$ (comonad comultiplication is $\Delta=F \eta I: G \rightarrow G G$, counit $\varepsilon: G \rightarrow{ }^{C}{ }^{e} \mathrm{M}$ is the counit of the adjunction). Denote ${ }^{\mathbb{G}} \mathrm{M}$ the Eilenberg-Moore category of $\mathbb{G}$-coalgebras, then ${ }^{\mathbb{G}} \mathrm{M}$ can be identified with ${ }^{H} \mathrm{M}$, since $G={ }^{e} H \square_{C^{e}}$. Also, the canonical forgetful functor $F_{G}:{ }^{\mathbb{G}} \mathrm{M} \rightarrow{ }^{e} \mathrm{M}$ can be identified with $F:{ }^{H} \mathrm{M} \rightarrow{ }^{C^{e}} \mathrm{M}$. 
By Prop. 2.1. of [17, the (strong) opmonoidal structure on $F$ implies a monoidal structure on the right adjoint $I$, and the adjunction is in the category of monoidal categories. This implies that the unit and counit are monoidal natural transformations. The following definition is tailor-made (see [13]):

Definition 4.1. Let $\langle\mathrm{M}, \square, I\rangle$ be a monoidal category. Then a bicomonad on $\mathrm{M}$ is a comonoid in the category of monoidal endofunctors from $M$ to $M$. Thus, it is an endofunctor $G: \mathrm{M} \rightarrow \mathrm{M}$, furnished with:

- a natural transformation $\kappa_{X, Y}:(G X) \square(G Y) \rightarrow G(X \square Y)$, and

- an arrow $\xi: C \rightarrow G C$

such that $\left\langle G, \kappa_{X, Y}, \xi\right\rangle$ is a monoidal functor;

- a natural transformation $\delta_{X}: G X \rightarrow G G X$ and

- a natural transformation $\varepsilon_{X}: G X \rightarrow X$

such that $\langle G, \delta, \varepsilon\rangle$ is a comonoid in $\mathrm{M}^{\mathrm{M}}$, and four compatibility axioms stating that $\delta$ is monoidal,

$$
\begin{aligned}
\delta_{X \otimes Y} \circ \kappa_{X, Y} & =\left(G \kappa_{X, Y} \circ \kappa_{G X, G Y}\right) \circ\left(\delta_{X} \otimes \delta_{Y}\right) \\
\delta_{I} \circ \xi & =G \xi \circ \xi
\end{aligned}
$$

and that $\varepsilon$ is monoidal

$$
\begin{gathered}
\varepsilon_{X \otimes Y} \circ \kappa_{X, Y}=\varepsilon_{X} \otimes \varepsilon_{Y} \\
\varepsilon \circ \xi=I
\end{gathered}
$$

Proposition 4.2. The endofunctor $G=F I={ }^{e} H \square_{C^{e}}$ : ${ }^{C}{ }^{e} \mathrm{M} \rightarrow{ }^{e} \mathrm{M}$ is a monoidal comonad with the structure maps:

$$
\begin{aligned}
& \delta_{X}: H \square_{C^{e}} X \rightarrow H \square_{C^{e}}\left(H \square_{C^{e}} X\right) \\
& h \otimes x \mapsto h_{(1)} \otimes\left(h_{(2)} \otimes x\right) \\
& \varepsilon_{X}: H \square_{C^{e}} X \rightarrow X \\
& h \otimes x \mapsto \varepsilon_{H}(h) x \\
& \kappa_{X, Y}:\left(H \square_{C^{e}} X\right) \square_{C}\left(H \square_{C^{e}} Y\right) \rightarrow H \square_{C^{e}}\left(X \square_{C} Y\right) \\
& \quad(h \otimes x) \otimes\left(h^{\prime} \otimes y\right) \mapsto h h^{\prime} \otimes(x \otimes y) \\
& \xi: C \rightarrow H \square_{C^{e}} C \\
& c \mapsto \eta(c)_{(1)} \otimes \alpha\left(\eta(c)_{(2)}\right)
\end{aligned}
$$

Proof. The associativity of $\kappa$ corresponds to the associativity of the multiplication $\mu$ of $H$, and $\xi$ is a unit for $\kappa$ precisely because $\eta$ is a unit for $\mu$. The monoidality of $\delta_{X}$ and $\varepsilon_{X}$ are due to the multiplicativity and unitalness of $\Delta_{H}$ and $\varepsilon_{H}$. Finally, $G$ is a comonad because $H$ is a coalgebra.

We now return to the question of dualizing Schauenburg's theorem. The original proof relies heavily on the fact that a left bialgebroid $A$ is a generator in the category ${ }_{A} \mathrm{M}$. This allows us to express the coproduct of $A$ as the action on $1_{A} \otimes 1_{R}$, $\Delta_{A}: a \mapsto a_{(2)} \otimes_{R} a_{(2)}:=a \cdot\left(1_{A} \otimes_{R} 1_{A}\right)$. An application of this reasoning seems impossible. Consider, however, the following monadic reformulation of the problem. A monoidal structure on ${ }^{H} \mathrm{M}$ such that $F:{ }^{H} \mathrm{M} \rightarrow{ }^{C} \mathrm{M} \simeq{ }^{C} \mathrm{M}^{C}$ is strict monoidal 
implies that the monoidal product on ${ }^{C^{e}} \mathrm{M}$ is lifted to the Eilenberg-Moore category of $\mathbb{G}$-coalgebras in the following sense:

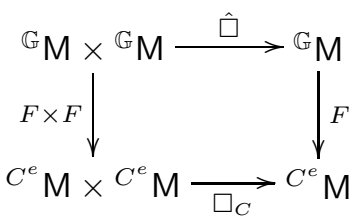

This is a special case of the problem of 'liftings of functors', orginally considered by Johnstone ([7]). Our reference is [20] (this volume), from which we quote part (1) of Theorem 3.3.

Theorem 4.3. Let $\mathbb{G}=\langle G, \delta, \varepsilon\rangle$ and $\mathbb{G}^{\prime}=\left\langle G^{\prime}, \delta^{\prime}, \varepsilon^{\prime}\right\rangle$ be comonads on the categories $\mathrm{M}$ and $\mathrm{M}^{\prime}$, respectively, and let $T: \mathrm{M}^{\prime} \rightarrow \mathrm{M}$ be a functor. Denote $U: \mathbb{G} \mathrm{M} \rightarrow \mathrm{M}$ and $U^{\prime}:{ }^{\mathbb{G}^{\prime}} \mathrm{M} \rightarrow \mathrm{M}^{\prime}$ the canonical forgetful functors.

Then, the liftings $\hat{T}: \mathbb{G}^{\prime} \mathrm{M} \rightarrow{ }^{\mathbb{G}} \mathrm{M}$ of $T$, in the sense:

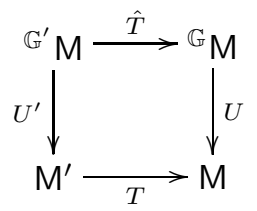

are in bijective correspondance with natural transformations $\kappa: T G^{\prime} \rightarrow G T$ for which the following diagrams commute:
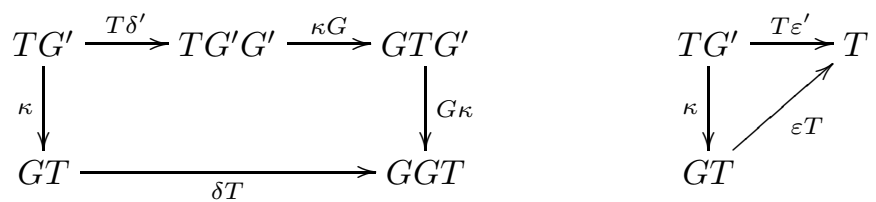

Taking $\mathrm{M}^{\prime}={ }^{C^{e}} \mathrm{M} \times{ }^{C^{e}} \mathrm{M}, \mathrm{M}={ }^{C^{e}} \mathrm{M}$ and $T={ }_{-} \square_{C}{ }_{-}{ }^{C^{e}} \mathrm{M} \times{ }^{C^{e}} \mathrm{M} \rightarrow{ }^{C^{e}} \mathrm{M}$, we find that liftings of the monoidal structure to ${ }^{\mathbb{G}} \mathrm{M} \simeq{ }^{H} \mathrm{M}$ are in bijective correspondance with natural transformations

$$
\kappa_{M, N}:\left(H \square_{C^{e}} M\right) \square_{C}\left(H \square_{C^{e}} N\right) \rightarrow H \square_{C^{e}}\left(M \square_{C} N\right)
$$

inducing commutative diagrams

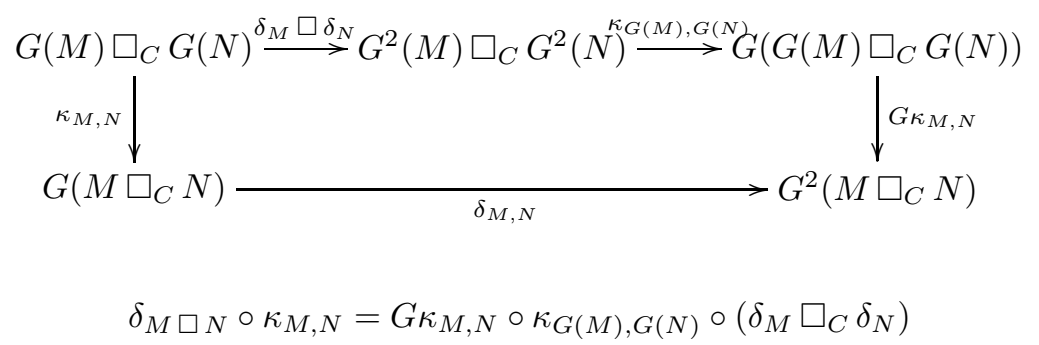

$$
\delta_{M \square N} \circ \kappa_{M, N}=G \kappa_{M, N} \circ \kappa_{G(M), G(N)} \circ\left(\delta_{M} \square_{C} \delta_{N}\right)
$$

and 


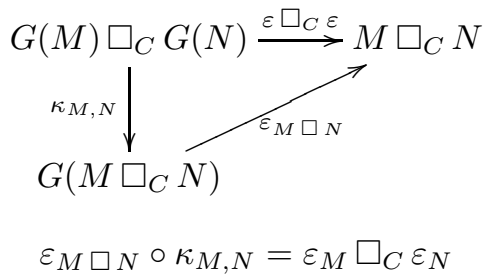

$$
\varepsilon_{M \square N} \circ \kappa_{M, N}=\varepsilon_{M} \square_{C} \varepsilon_{N}
$$

The two diagrams above recover two of the compatibility relations 4.1 and 4.3) of a bicomonad. If, furthermore, we have an arrow $\xi: C \rightarrow G(C)$ making $C$ a $\mathbb{G}$-coalgebra such that the remaining two bicomonad conditions 4.2 and 4.4) are satisfied, then ${ }^{\mathbb{G}} \mathrm{M}$ becomes a (unital) monoidal category. Summarizing, we have the following weakened form of Schauenburg's theorem:

Theorem 4.4. Let $\langle H, \bar{\Delta}, \bar{\varepsilon}\rangle$ be a comonoid in ${ }^{C^{e}} \mathrm{M}$. Then there is a bijective correspondance between

(1) monoidal structures on ${ }^{H} \mathrm{M}$ such that the forgetful functor $F:{ }^{H} \mathrm{M} \rightarrow{ }^{e} \mathrm{M}$ is strict monoidal

(2) a map $\kappa_{M, N}:\left(H \square_{C^{e}} M\right) \square_{C}\left(H \square_{C^{e}} N\right) \rightarrow H \square_{C^{e}}\left(M \square_{C} N\right)$, natural in both arguments and a map $\xi: C \rightarrow H \square_{C^{e}} C$ such that $\langle H, \bar{\Delta}, \bar{\varepsilon} ; \kappa, \xi\rangle$ constitutes a bicomonad, i.e. the compatibilty conditions 4.1, 4.2, 4.3 and 4.4 are satisfied.

Notice that in proving Theorem 2.7, we established (1) by constructing the maps $\kappa$ and $\xi$ of (2) from bicoalgebroid structure maps. For bialgebroids, a stronger result can be proved because a bialgebroid structure not only implies, but is equivalent to, the analogue of (2).

We now turn to the scalar extension of bicoalgebroids to investigate it from a comonadic point of view. A scalar extension $H^{\prime}=D \sharp H$ of the bicoalgebroid $H$ by the BCC $D$ gives rise to an adjunction between the respective comodule categories. The forgetful functor $F^{\prime}:{ }^{D \sharp H} \mathrm{M} \rightarrow{ }^{H} \mathrm{M}$ is induced by the epi $\left(\varepsilon_{D} \otimes H\right): D \sharp H \rightarrow$ $H$. It has the right adjoint induction functor

$$
I^{\prime}:{ }^{H} \mathrm{M} \rightarrow{ }^{D \sharp H} \mathrm{M}, \quad X \mapsto(D \sharp H) \square_{H} X
$$

with the unit and counit of the adjunction being

$$
\begin{aligned}
& v: X \rightarrow I F(X)=D \sharp H \square_{H} X, \quad x \mapsto \delta_{D \sharp H}(x) \\
& \tau: F I(Y)={ }^{H}(D \sharp H) \square_{H} Y \rightarrow Y, \quad(d \otimes h) \otimes y \mapsto \varepsilon_{D}(d) \varepsilon(h) y
\end{aligned}
$$

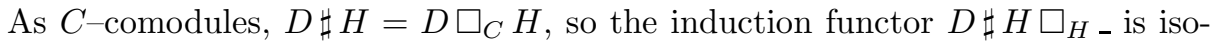
morphic to $D \square_{C}$. . By Lemma 3.5, this functor is strong monoidal, hence also opmonoidal. It will remain opmonoidal upon composition with the opmonoidal forgetful functor, making the canonical comonad $\mathbb{G}=\left\langle F^{\prime} I^{\prime}, F^{\prime} v I^{\prime}, \tau\right\rangle$ an opmonoidal endofunctor. The compatibility of the opmonoidal and comonadic structure make $\mathbb{G}$ an opmonoidal comonad, not to be confused with the monoidal comonad which we have christened 'bicomonad' earlier. We state the definition as concisely as possible.

Definition 4.5. An opmonoidal comonad $\left\langle\left\langle G, G_{X, Y}, G^{0}\right\rangle, \Delta, \varepsilon\right\rangle$ on a monoidal category $\langle\mathcal{C}, \square, \iota\rangle$ consists of 
- An opmonoidal endofunctor $\left\langle G, G_{X, Y}, G^{0}\right\rangle$ on $\mathrm{M}$ and

- a comonad $\langle G, \Delta, \varepsilon\rangle$

such that $\Delta$ and $\varepsilon$ are opmonoidal

Proposition 4.6. Let $D$ be a $B C C$ over the left bicoalgebroid $H$. Then the endofunctor $G=D \square_{C-}$ is an opmonoidal comonad on ${ }^{H} \mathrm{M}$.

Proof. Recall that $G_{X, Y}: D \square(X \square Y) \rightarrow(D \square X) \square(D \square Y)$ reads, on elements: $d \otimes x \otimes y \mapsto d_{(1)} \otimes x^{\langle-1\rangle} \otimes d_{(2)} \triangleleft x^{\langle-1\rangle} \otimes y$ and $G^{0}=\left(\varepsilon_{D} \square C\right): D \square C \rightarrow C$. The comonad structure follows from the coalgebra structure of $D$.

We have only to check the compatibility of the comonad and opmonoidal structure, meaning four commutative diagrams. Opmonoidality of the comultiplication means (1):

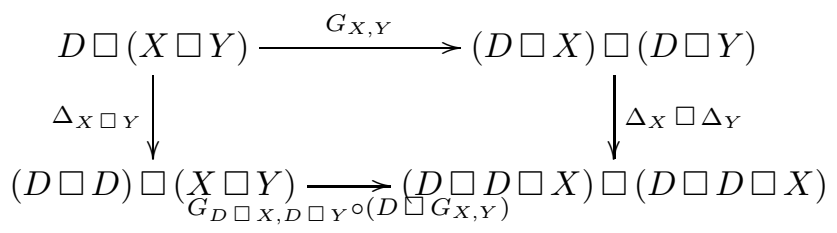

An easy calculation shows the commutativity of the diagram. The upper and right hand side map compose to give

$$
\begin{aligned}
& d \otimes x \otimes y \mapsto d_{(1)} \otimes\left(d_{(2)(1)} \otimes x^{\langle 0\rangle}\right) \otimes\left(d_{(2)(2)} \triangleleft x^{\langle-1\rangle} \otimes y\right) \mapsto \\
& \quad \mapsto d_{(1)(1)} \otimes\left(d_{(2)(1)} \otimes x^{\langle 0\rangle}\right)^{\langle 0\rangle} \otimes d_{(1)(2)} \triangleleft\left(d_{(2)(1)} \otimes x^{\langle 0\rangle}\right)^{\langle-1\rangle} \otimes d_{(2)(2)} \triangleleft x^{\langle-1\rangle} \otimes y
\end{aligned}
$$

Using the braided cocommutativity of $D$, we have:

$$
\begin{aligned}
& d_{(1)(1)} \otimes d_{(2)(1)}{ }^{\langle 0\rangle} \otimes x^{\langle 0\rangle\langle 0\rangle} \otimes d_{(1)(2)} \triangleleft\left(d_{(2)(1)}{ }^{\langle-1\rangle} x^{\langle 0\rangle\langle-1\rangle}\right) \otimes d_{(2)(2)} \triangleleft x^{\langle-1\rangle} \otimes y= \\
& =d_{(1)(1)} \otimes d_{(1)(2)} \otimes x^{\langle 0\rangle\langle 0\rangle} \otimes d_{(2)(1)} \triangleleft x^{\langle 0\rangle\langle-1\rangle} \otimes d_{(2)(2)} \triangleleft x^{\langle-1\rangle} \otimes y,
\end{aligned}
$$

which is the composition of the lower and left hand side maps. The second diagram (2) for the opmonoidality of $\Delta$ is

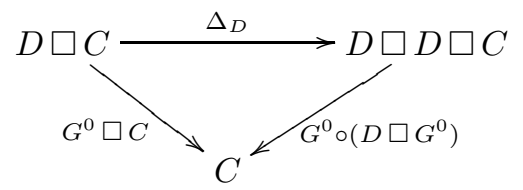

which commutes by the counit property of $\varepsilon_{D}\left(G^{0}=\varepsilon_{D} \square C\right)$. The remaining two diagrams stating the opmonoidality of $\varepsilon_{X}: D \square X \rightarrow X$ are (3)

$$
\left(\varepsilon_{X} \square \varepsilon_{Y}\right) \circ G_{X, Y}=\varepsilon_{X, Y},
$$

commuting since $\varepsilon\left(d_{(1)}\right) x^{\langle 0\rangle} \square_{C} \varepsilon\left(d_{(2)} \triangleleft x^{\langle-1\rangle}\right) y=\varepsilon(d) x \square_{C} y$, and (4)

$$
\varepsilon_{C}=G^{0}
$$

which is a triviality. 
We briefly recall the dual situation, for scalar extensions of bialgebroids (for details, see [1]). For a scalar extension $Q \# H$ of a (left) bialgebroid $H$ over $R$, the inclusion $\iota: H \hookrightarrow Q \# H$ induces a monoidal forgetful functor $U:{ }_{Q \# H} \mathrm{M} \rightarrow{ }_{H} \mathrm{M}$. The left adjoint of $U$ is

$$
I:{ }_{H} \mathrm{M} \rightarrow{ }_{Q \# H} \mathrm{M}, \quad X \mapsto(Q \# H) \otimes_{H} X
$$

with unit and counit

$$
\begin{aligned}
& \eta: X \rightarrow U I(X)={ }_{H}(Q \# H) \otimes_{H} X, \quad x \mapsto\left(1_{Q} \otimes 1_{H}\right) \otimes x \\
& \varepsilon: I U(Y)={ }_{Q \# H}(Q \# H) \otimes_{H} Y \rightarrow Y, \quad(q \otimes h) \otimes y \mapsto(q \# h) \triangleright y=q \cdot(h \triangleright y)
\end{aligned}
$$

Note that $U=\operatorname{Hom}_{H-}\left(H,{ }_{-}\right)$. By Prop. 4.7 of [1], $I$ is strong monoidal, so the underlying endofunctor of the canonical monad $\mathbb{T}=\langle U I, U \varepsilon I, \eta\rangle$ on ${ }_{H} \mathrm{M}$ will be monoidal, being the composition of two monoidal functors. Thus, the scalar extension of bialgebroids gives rise to a monoidal monad on the module category of the 'smaller' bialgebroid.

Having seen that scalar extensions of bialgebroids and bicoalgebroids by BCA's and BCC's give rise to monoidal monads and opmonoidal comonads, respectively, in the rest of this paper, we make some tentative steps in the reverse direction.

First, note that any monoidal category $\langle\mathcal{C}, \square, I\rangle$ may be embedded (monoidally, but not fully) into the category of it's endofunctors ([5]), which is monoidal with the composition of functors as monoidal product and the identity functor as monoidal unit. The inclusion is given by $\mathcal{C} \hookrightarrow \mathcal{C}^{\mathcal{C}}, X \mapsto \hat{X} \square$ _, and the image of the inclusion will be denoted $\hat{\mathcal{C}}$. The arrows of $\hat{\mathcal{C}}$ are natural transformations of the form $\alpha_{Z}=\alpha \square Z: X \square Z \rightarrow Y \square Z$, with $\alpha: X \rightarrow Y$ an arrow in $\mathcal{C}$. An immediate consequence is that for any map $\gamma \in \mathcal{C}$ :

$$
\gamma_{X \square Y}=\gamma_{X} \square Y,
$$

since $\gamma_{X \square Y}=(\gamma \square X) \square Y=\gamma_{X} \square Y$.

Proposition 4.7. Let $\mathcal{C}$ be a monoidal category, and $\left\langle D \square_{-}, D_{X, Y}, D^{0}\right\rangle$ an opmonoidal endofunctor in $\hat{\mathcal{C}}$. Then there is a natural transformation (between endo-

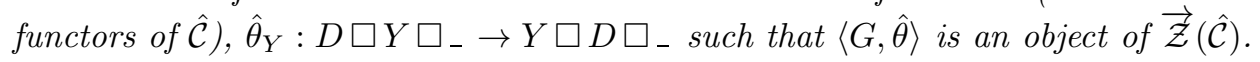

Proof. We shall only prove the latter statement, which amounts to constructing a natural transformation

$$
\hat{\theta}_{Y}(-):(D \square Y) \square \_\rightarrow(Y \square D) \square \_
$$

satisfying eqs. 3.5 and 3.6. It is easily verified that

$$
\begin{aligned}
\hat{\theta}_{Y}(X):(D \square Y) \square X & \rightarrow(D \square Y) \square(D \square X) \rightarrow(Y \square D) \square X \\
\hat{\theta}_{Y}(X) & =\left(G^{0} \square 1\right) \circ G_{Y, X}
\end{aligned}
$$

is appropriate. Eq. 3.5 means that the following diagram commutes (suppressing natural isomorphisms): 


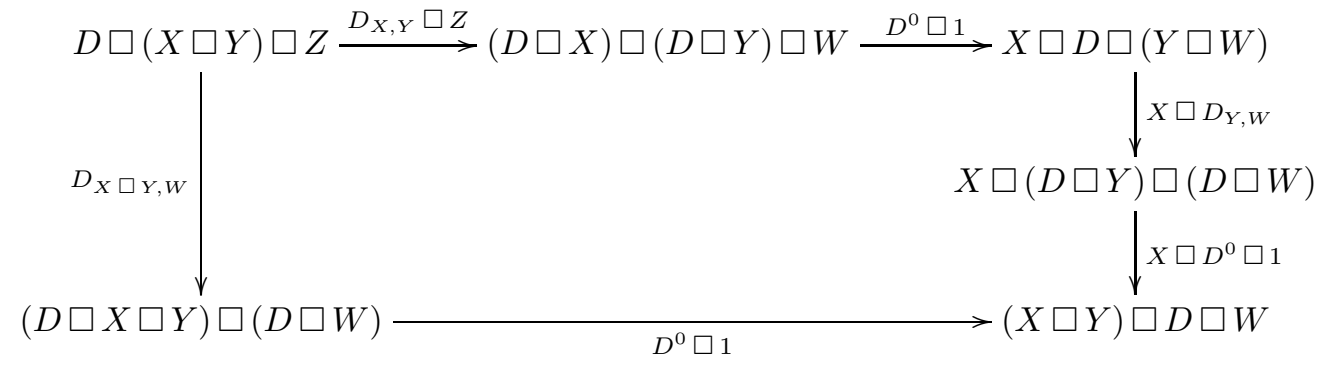

The diagram commutes by the coassociativity of $D_{X, Y}$ and because $D^{0}$ is a counit for $D_{X, Y}$. Note that because $D_{X, Y}=D_{X, \iota \square Y}=D_{X, \iota} \square Y$, the counit relation for $D^{0}$ is equivalent to the following property for $D_{X, Y}$ :

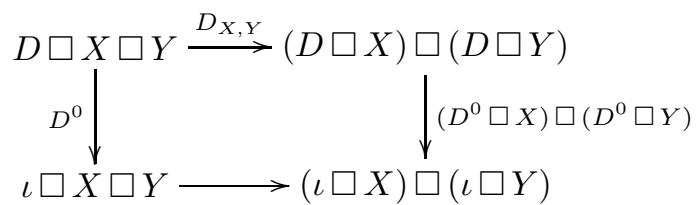

Remark 4.8. Clearly, by the embedding $\mathcal{C} \hookrightarrow \hat{\mathcal{C}}$, we have in fact proven that $D$ is an object in $\overrightarrow{\mathcal{Z}}(\mathcal{C})$.

Remark 4.9. Entirely analogously, for a monoidal category $\langle\mathrm{M}, \otimes, \iota\rangle$, and a monoidal endofunctor $\left\langle T, T_{X, Y}, T_{0}\right\rangle$ in $\hat{\mathrm{M}}$, of the form $T=Q \otimes$ _ the natural transformation

$$
\begin{aligned}
\hat{\bar{\theta}}_{Y}(X): Y \otimes Q \otimes X & \rightarrow Q \otimes Y \otimes Q \otimes X \rightarrow Q \otimes Y \otimes X \\
\hat{\bar{\theta}}_{Y}(X) & =T_{X, Y} \circ\left(T_{0} \otimes 1\right)
\end{aligned}
$$

makes $\left\langle T, \hat{\bar{\theta}}_{Y}\right\rangle$ an object in $\overleftarrow{\mathcal{Z}}(\mathrm{M})$

We saw that for a BCC $D \in{ }^{H} \mathcal{Y} \mathcal{D}_{H}$ over a bicoalgebroid, $D \square_{C}$ - is not only an opmonoidal endofunctor, but an opmonoidal comonad. Unfortunately, it seems unlikely that the correspondence between opmonoidal endofunctors of $\hat{\mathcal{C}}$ and objects of $\overrightarrow{\mathcal{Z}}(\mathcal{C})$ can be extended to a correspondence between opmonoidal comonads of $\hat{\mathcal{C}}$ and BCC's in $\overrightarrow{\mathcal{Z}}(\mathcal{C})$ without further assumptions.

\section{ACKNOWLEDGMENTS}

I am indebted to Kornél Szlachányi for his continued interest and our discussions, especially concerning the cocenter construction of 2.2. I'm thankful also to Gabi Böhm for her comments and for calling refs. 77 and 20 to my attention. The suggestions of the Referee helped much to clarify the exposition.

\section{REFERENCES}

[1] I. Bálint, K. Szlachányi, Finitary Galois extensions over noncommutative bases, J. Algebra 296 (2006) 520-560

[2] T. Brzeziński, G. Militaru, Bialgebroids, $\times_{A}$-bialgebras and duality, J. Algebra 251 (2002) 279-294 
[3] T. Brzeziński, R. Wisbauer, Corings and Comodules, London Math. Soc. LNS 309, Cambridge Univ. Press 2003

[4] S. Caenepeel, Dingguo Wang, Yanmin Yin, Yetter-Drinfeld modules over weak Hopf algebras and the center construction, Ann. Univ. Ferrara - Sez. VII - Sc. Mat. 51 (2005), 69-98.

[5] A. Joyal, R.H. Street, Braided tensor categories, Advances in Mathematics 102, 20-78 (1993)

[6] A. Joyal, R.H. Street, Tortile Yang-Baxter operators in tensor categories, J. Pure Appl. Algebra 71, 43-51 (1991)

[7] Johnstone, P.T., Adjoint lifting theorems for categories of modules, Bull. Lond. Mat. Soc. 7, 294-297 (1975)

[8] L. Kadison, K. Szlachányi, Bialgebroid actions on depth two extensions and duality, Advances in Mathematics 179 (2003) 75-121

[9] L. Kadison, Co-depth two and related topics, math.QA/0601001

[10] C. Kassel, "Quantum Groups", Grad. Textsin Math. 155, Springer Verlag, Berlin, 1995

[11] S. Mac Lane, Categories for the Working Mathematician, 2nd edition, GTM 5, Springer-Verlag New-York Inc., 1998

[12] S. Majid, Representations, duals and quantum doubles of monoidal categories, Rend. Circ. Mat. Palermo (2) Suppl. No. 26 (1991), 197-206

[13] I. Moerdijk, Monads on tensor categories, Journal of Pure and Applied Algebra, 168 (2002) 189-208

[14] S. Montgomery, Hopf Algebras and Their Actions on Rings, CBMS Lecture Notes 82, Am. Math. Soc., Providence, RI (1993)

[15] P. Schauenburg, Duals and doubles of quantum groupoids in "New trends in Hopf algebra theory", Contemporary Mathematics 267, p. 273, AMS 2000

[16] Hans-Jürgen Schneider, Principal homogeneous spaces for arbitrary Hopf algebras, Isr. J. of Mathematics, Vol. 72, Nos. 1-2. (1990)

[17] K. Szlachányi, The monoidal Eilenberg-Moore construction and bialgebroids, Journal of Pure and Applied Algebra 182 (2003) 287-315

[18] M. Takeuchi, Morita theorems for categories of comodules, J. Fac. Sci. Univ. Tokyo, Sec. IA, 24, (1977) 629-644

[19] B. Torrecillas, F. van Oystaeyen, Y. H. Zhang, The Brauer Group of a Cocommutative Coalgebra, Journal of Algebra, 177 (1995) 536-568

[20] Robert Wisbauer, Algebras versus coalgebras, to appear in Applied Categorical Structures (this volume)

Research Institute for Particle and Nuclear Physics, Budapest

E-mail address: balint@rmki.kfki.hu 